Engineering Physics and Mathematics Division

\title{
INPUT RELEGATION CONTROL FOR GROSS MOTION OF A KINEMATICALLY REDUNDANT MANIPULATOR
}

\author{
M. A. Unseren
}

ORNL/TM--12165

DE93 002735

DATE PUBLISHED - October 1992

Office of Engineering Research Program Basic Energy Sciences and

Office of Technology Support Programs Office of Nuclear Energy

Prepared by the

OAK RIDGE NATIONAL LABORATORY

Oak Ridge, Tennessee 37831 managed by

MARTIN MARIETTA ENERGY SYSTEMS, INC. for the U.S. DEPARTMENT OF ENERGY under contract DE-AC05-84OR21400

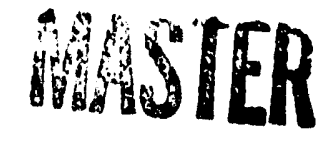




\section{CONTENTS}

ABSTRACT . . . . . . . . . . . . . . . . . . . $\mathrm{v}$

1. INTRODUCTION . . . . . . . . . . . . . . . . . . . . 1

2. PROBLEM STATEMENT AND SYSTEM DESCRIPTION . . . . 3

2.1 SYSTEM VARIABLES AND COORDINATE FRAMES . . . . . 3

2.2 KINEMATIC MANIPULATOR MODEL . . . . . . . . . 3

2.3 DYNAMIC MANIPULATOR MODEL . . . . . . . . . . 5

3. RESOLUTION OF KINEMATIC REDUNDANCY . . . . . . . . 7

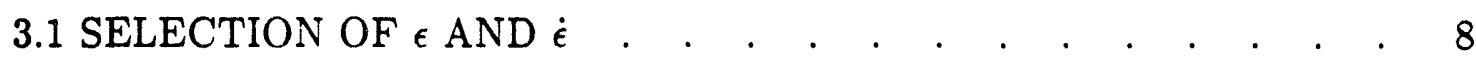

3.2 SELECTION OF $B$ SUCH THAT $\epsilon$ IS A SUBSET OF $\dot{q}$. $\quad$. $\quad$. $\quad . \quad 9$

3.3 CHOOSING $B$ ORTHOGONAL TO THE ROWS OF $J$. . . . . 13

3.4 SELECTING $B$ BY APPLICATION OF THE ZERO-EIGENVALUE MATRIX THEOREM . . . . . . . . . . . . . . 16

3.5 THE VECTOR CROSS PRODUCT METHOD FOR PLANAR MANIPULATORS . . . . . . . . . . . . . . 17

4. RIGID BODY DYNAMICAL MODEL AND CONTROL ARCHITECTURE 23

5. CONCLUSION AND FUTURE WORK . . . . . . . . . . . 25

6. ACKNOWLEDGMENT . . . . . . . . . . . . . . . 27



APPENDIX B . . . . . . . . . . . . . . . . . . 31

APPENDIX C . . . . . . . . . . . . . . . . . . 39

REFERENCES . . . . . . . . . . . . . . . . . . 41 


\section{LIST OF FIGURES}

Fig.

$\underline{\text { Page }}$

1 System configuration and coordinate system assignment . . . . 4

2 System configuration and coordinate system assignment for the CESARm research manipulator . . . . . . . . . . , 11

3 Planar revolute redundant manipulator . . . . . . . . . . 18 


\begin{abstract}
This report proposes a method for resolving the kinematic redundancy of a serial link manipulator moving in a three-dimensional workspace. The underspecified problem of solving for the joint velocities based on the classical kinematic velocity model is transformed into a well-specified problem. This is accomplished by augmenting the original model with additional equations which relate a new vector variable quantifying the redundant degrees of freedom (DOF) to the joint velocities. The resulting augmented system yields a well specified solution for the joint velocities. Methods for selecting the redundant DOF quantifying variable and the transformation matrix relating it to the joint velocities are presented so as to obtain a minimum Euclidean norm solution for the joint velocities. The approach is also applied to the problem of resolving the kinematic redundancy at the acceleration level. Upon resolving the kinematic redundancy, a rigid body dynamical model governing the gross motion of the manipulator is derived. A control architecture is suggested, which according to the model, decouples the Cartesian space DOF and the redundant DOF.
\end{abstract}




\section{INTRODUCTION}

The historical trend of minimality in design is manifested in the majority of present day robotic manipulators including their control systems [1]. It is argued in [1] that this historical trend has impeded, to some extent, the development of new theory and new computational and analytical tools needed for the design of future robotic systems. Two of the attributes proposed in [1] for the design and control of future robots are the enlargement of the input and state spaces of the manipulator such that it has a larger number of degrees of freedom (DOF) than the minimum required. The additional DOF enable several tasks to be accomplished simultaneously during motion of the manipulator. The concept of relegation of control which involves assigning specific tasks to subsets of the control inputs to a manipulator system with enlarged state and input spaces is proposed as a particular type of generalized, nonlinear decoupling control in [1]. The control philosophy framework [1] is presented in a conceptual, qualitative manner.

A kinematically redundant manipulator $[2,3,4,5,6]$ is a classical example of an actuated mechanical system possessing enlarged input and state spaces. This report formalizes analytically and mathematically the input relegation control philosophy [1] to the decoupling of the nonredundant and redundant DOF during gross motion of a kinematically redundant manipulator. The approach to resolving the kinematic redundancy as well as deriving a rigid body dynamical model and a control architecture for the manipulator are based on a general method for modeling and controlling constrained mechanical systems $[7,8,9]$.

In the previous work $[7,8,9]$, the motion of the dynamical system studied was restricted by a set of $k$ bilateral constraint equations imposed on $n$ generalized velocities which describe the configuration of the system, where $k<n$. Thus the problem of solving the constraint equations to obtain generalized velocities which satisfy the constraints is underspecified and there are infinitely many solutions. $(n-k)$ new scalar variables (termed pseudovelocities in $[7,9])$ consisting of linear combinations of the generalized velocities were introduced, which, together with the original constraints, yielded a well specified solution for the generalized velocities.

The Jacobian matrix which transforms the joint velocities to obtain the Cartesian velocities of a point, link, or end effector located on a kinematically redundant manipulator is rectangular with fewer rows than columns. Thus the problem of solving the kinematic velocity transformation equations for the joint velocities is underspecified and there are infinitely many solutions. Interestingly, the problems of resolving the redundancy of a manipulator and of determining generalized velocities which satisfy the constraint equations in a constrained mechanical system appear to be intimately related. It will be investigated here if the approach in $[7,8,9]$ can be extended to the problem of modeling a kinematically redundant manipulator.

The report is organized as follows. A general procedure for resolving the kinematic redundancy is first presented. Several techniques for applying the procedure are discussed. A rigid body dynamical model is derived which consists of separate equations of motion for the nonredundant and redundant DOF. Finally, a decoupled control architecture is developed based on the separated form of the model. 


\section{PROBLEM STATEMENT AND SYSTEM DESCRIPTION}

The problem is to resolve the kinematic redundancy of a serial-link manipulator moving in a three-dimensional workspace. The manipulator has a stationary base and contains $N$ single DOF joints. Upon resolving the kinematic redundancy, a rigid body dynamical model and a control architecture are developed. The kinematical and dynamical models of the redundant manipulator are assumed to be known. The configuration of the system is shown in Fig. 1.

\subsection{SYSTEM VARIABLES AND COORDINATE FRAMES}

The joint positions of the manipulator are the generalized coordinates describing the configuration of the system. The system variables include the generalized coordinates, velocities, accelerations, and the generalized input forces (i.e., the joint torques) applied to the joint actuators.

As shown in Fig. 1, the coordinate frame $\left(X_{k}, Y_{k}, Z_{k}\right)$ is assigned to the $k_{t h}$ link of the manipulator, where $k=0,1, \ldots, N$. The origin of the moving $\left(X_{N}, Y_{N}, Z_{N}\right)$ coordinate system is located at the centerpoint of the end effector. The $\left(X_{0}, Y_{0}\right.$, $Z_{0}$ ) coordinate frame is the stationary base reference system.

\subsection{KINEMATIC MANIPULATOR MODEL}

The kinematic model for a serial-link manipulator which relates the Cartesian velocities of a point, link, or end effector to the joint velocities is given by:

$$
\dot{x}=J(q) \dot{q} .
$$

The joint positions of the manipulator defined by the vector $q=\left[q_{1}, q_{2}, \ldots, q_{N}\right]^{T}$ are the generalized coordinates, where superscript $T$ denotes a transposition. The Cartesian velocities of a point, link, or end effector on the manipulator are defined by the $(M \times 1)$ vector $\dot{x}$. It is assumed that $M<N$. For the case where the Cartesian translational and rotational velocities of one of the rigid links (e.g., the $k=N$ th link is the rigid body end effector) are to be controlled, $M=6$ and $\dot{x}=\left[\left(v_{0}^{k}\right)^{T},\left(\omega_{0}^{k}\right)^{T}\right]^{T}$. The $(3 \times 1)$ vector $v_{0}^{k}$ is the translational velocity of the $k t h$ link at the point coinciding with the origin of the $\left(X_{k}, Y_{k}, Z_{k}\right)$ coordinate system. The $(3 \times 1)$ vector $\omega_{0}^{k}$ is the angular velocity of the kth link. $v_{0}^{k}$ and $\omega_{0}^{k}$ are expressed in the base coordinates. For the case where only the Cartesian translational velocity of a point on the manipulator is to be controlled, then $M=3$ and $\dot{x}=v_{0}^{k}$. In Eq. (1), $J(q)$ is the $(M \times N)$ manipulator Jacobian matrix. $J(q)$ is assumed to possess full rank $M$.

The corresponding kinematic manipulator model at the acceleration level is obtained by differentiating Eq. (1) in:

$$
\ddot{x}=J \ddot{q}+\dot{j} \dot{q} .
$$

The $(M \times N)$ matrix $\dot{J}=(\partial J / \partial q) \dot{q}$ in Eq. (2) is a function of $(q, \dot{q})$. 


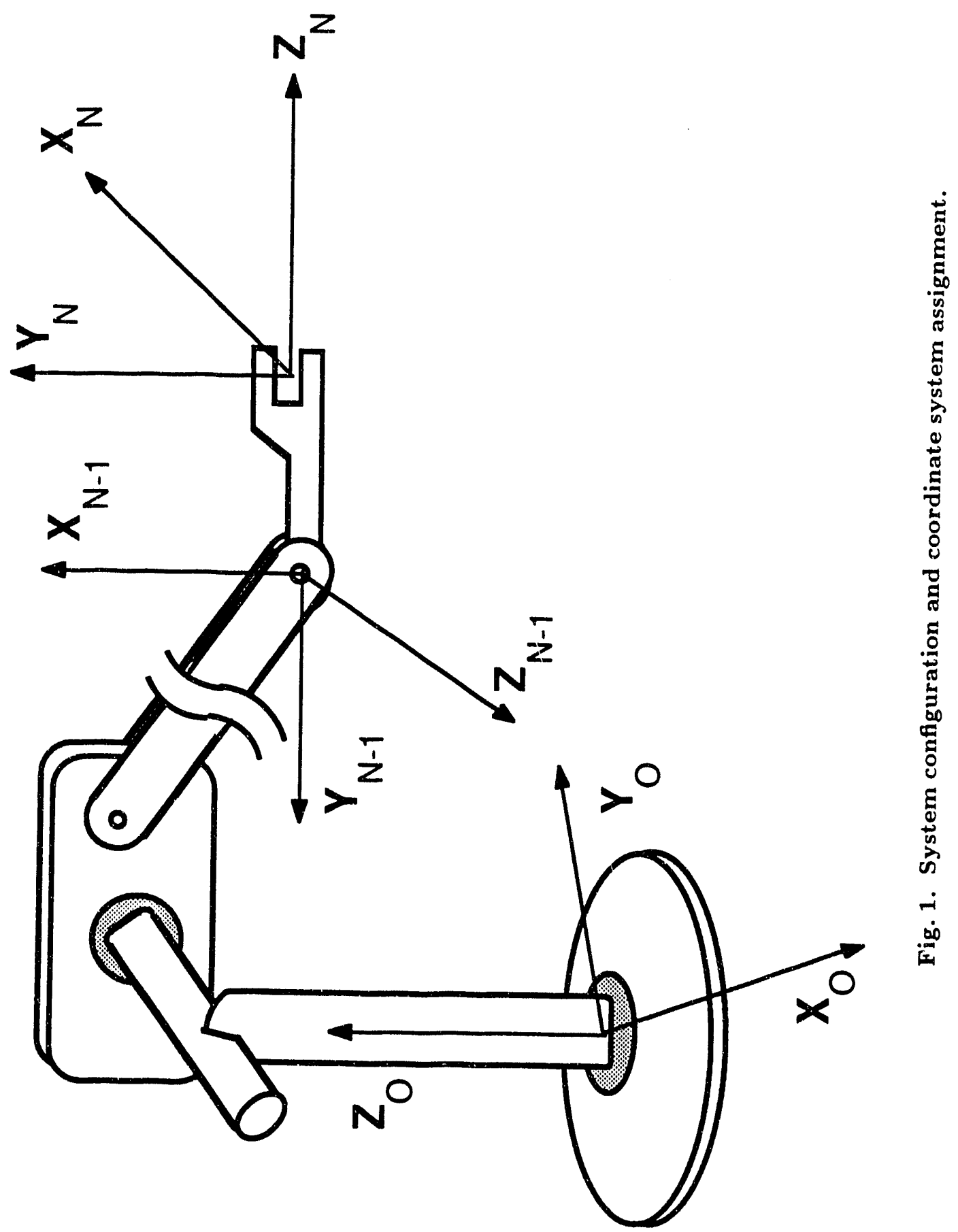




\subsection{DYNAMIC MANIPULATOR MODEL}

The dynamical model in the joint space for the manipulator is given in Lagrange's formulation by:

$$
\tau=D(q) \ddot{q}+C(q, \dot{q})+G(q) .
$$

The generalized input forces (i.e., the joint torques) are denoted by the vector $\tau=\left[\tau_{1}, \tau_{2}, \ldots, \tau_{N}\right]^{T}$. The $(N \times N)$ symmetric, positive definite, inertia matrix is $D(q)$, and the Coriolis and centripetal forces are described by the $(N \times 1)$ vector $C(q, \dot{q})$. The gravity forces acting on the manipulator are described by the $(N \times 1)$ vector $G(q)$.

The problem of resolving the kinematic redundancy based on the kinematic model given by Eq. (1) is discussed next. 


\section{RESOLUTION OF KINEMATIC REDUNDANCY}

The problem is to solve Eq. (1) for the generalized velocities $\dot{q}$ given the Cartesian velocities $\dot{x}$ and the generalized coordinates. The solution to the inverse kinematics is underspecified because the number of unknown generalized velocities $(N)$ exceeds the number of equations $(M)$ in the kinematic model. Equation (1) has infinitely many solutions for $\dot{q}$.

Motivated by the previous work $[7,8,9]$, a new vector variable $\epsilon=\left[\epsilon_{1}, \epsilon_{2}, \ldots, \epsilon_{N-M}\right]^{T}$ is introduced to resolve the kinematic redundancy. The number of scalar elements contained in $\epsilon$ is equal to the number of redundant DOF contained in the system, namely $(N-M)$. It is defined by:

$$
\epsilon=B(q) \dot{q}
$$

The $((N-M) \times N)$ matrix $B(q)$ in Eq. (4) is selected so that the composite $(N \times N)$ matrix $\left(J^{T}(q), B^{T}(q)\right)^{T}$ is nonsingular. It is convenient to partition the inverse of $\left(J^{T}(q), B^{T}(q)\right)^{T}$ into two matrices:

$$
\left[\begin{array}{c}
J(q) \\
B(q)
\end{array}\right]^{-1}=[\Pi(q), \Sigma(q)]
$$

whers $\Pi(q)$ is a $(N \times M)$ matrix and $\Sigma(q)$ a $(N \times(N-M))$ matrix. Equation (5) implies that $J \Pi=I_{M \times M}, J \Sigma=0_{(M \times(N-M))}, B \Pi=0_{((N-M) \times M)}$, $B \Sigma=I_{((N-M) \times(N-M))}$ and $(\Pi J+\Sigma B)=I_{N \times N}$. (Here $I_{r \times r}$ denotes an $(r \times r)$ identity matrix and $0_{r \times s}$ an $(r \times s)$ matrix of zeros). The choice of matrix $B(q)$ and vector $\epsilon$ by the designer is somewhat arbitrary. Several examples are provided later in this section.

Differentiating Eq. (4) establishes a relation between $\dot{\epsilon}$ and $\ddot{q}$ :

$$
\dot{\epsilon}=B \ddot{q}+\dot{B} \dot{q} \text {. }
$$

The $((N-M) \times N)$ matrix $\dot{B}=(\partial B / \partial q) \dot{q}$ in Eq. (6) is a function of $(q, \dot{q})$.

Equations (1) and (4) can be solved for $\dot{q}$, and Eqs. (2) and (6) for $\ddot{q}$ :

$$
\begin{gathered}
\dot{q}=\Pi \dot{x}+\Sigma \epsilon \\
\ddot{q}=\Pi \ddot{x}+\Sigma \dot{\epsilon}-(\Pi \dot{J}+\Sigma \dot{B})(\Pi \dot{x}+\Sigma \epsilon)
\end{gathered}
$$

in which Eq. (5) has been invoked. In Eqs. (7) and (8), $\dot{q}$ and $\ddot{q}$ have been expressed as functions of the variables $\{\dot{x}, \epsilon, q\}$ and $\{\dot{x}, \ddot{x}, \epsilon, \dot{\epsilon}, q\}$, respectively. Substituting the right hand side of Eq. (7) into the kinematic velocity model (Eq. 1) reveals that the expression $(J \Sigma \epsilon)$ identically vanishes regardless of the value of $\epsilon$, since $(J \Sigma)=0_{(M \times(N-M))}$. Likewise, substituting the right hand side of Eq. (7) into Eq. (4) reveals that the expression $(B \Pi \dot{x})$ identically vanishes regardless of the value of $\dot{x}$, since $(B \Pi)=0_{((N-M) \times M)}$. Therefore the physical motion of the point, link, or end effector being controlled in Cartesian space $\{\dot{x}\}$ is independent of vector $\epsilon$. 
The preceding general development demonstrates that it is possible to produce equations of the form of Eqs. (7) and (8) by introducing additional Eqs. (4) and (6) which quantify the behavior of the redundant DOF. However, it does not present a criteria for selecting $\epsilon$. Moreover, the development does not indicate an operational procedure for determining matrix $B(q)$. This matrix is not unique, and several methods for constructing it as well as a method for determining $\epsilon$ are discussed next.

\subsection{SELECTION OF $\epsilon$ AND $\dot{\epsilon}$}

The achievement or fulfillment of additional tasks or goals other than controlling the motion of a point or link on the manipulator (i.e., $\dot{x}$ ) to track a reference trajectory will be relegated to the $(N-M)$ redundant DOF. The specific additional task or goal is pertinent to the selection of $\epsilon$ and $\dot{\epsilon}$. To illustrate the quantification of the redundant DOF, analytical expressions for $\epsilon$ and $\dot{\epsilon}$ are developed in this section which lead to minimum Euclidean norm solutions for the generalized velocities and accelerations, respectively. Starting with redundancy resolution at the velocity level, $\epsilon$ is selected to minimize $\|\dot{q}\|^{2}$ using Eq. (7):

$$
\|\dot{q}\|^{2}=(\Pi \dot{x}+\Sigma \epsilon)^{T}(\Pi \dot{x}+\Sigma \epsilon) .
$$

Taking the partial derivative of Eq. (9) with respect to $\epsilon$ and equating the result to zero give:

$$
\Sigma^{T} \Sigma \epsilon+\Sigma^{T} \Pi \dot{x}=0_{((N-M) \times 1)} .
$$

Since $\Sigma$ has full rank $(N-M)$, then matrix $\left(\Sigma^{T} \Sigma\right)$ is positive definite, symmetric, and therefore nonsingular. Thus Eq. (10) can be solved for $\epsilon$ :

$$
\epsilon=-\left(\Sigma^{T} \Sigma\right)^{-1} \Sigma^{T} \Pi \dot{x}
$$

It is important to note that matrices $\Pi$ and $\Sigma$ are unknown quantities whereas $\dot{x}$ is known on the right-hand side of Eq. (11). To determine $\epsilon$ using Eq. (11), the designer first chooses a matrix $B(q)$ which immediately leads to the determination of $[\Pi(q), \Sigma(q)]$ by Eq. (5). Sufficient information is now available to calculate $\epsilon$. Substituting the right-hand side of Eq. (11) into Eq. (7) and grouping terms give:

$$
\dot{q}=\left(I_{N \times N}-\Sigma\left(\Sigma^{T} \Sigma\right)^{-1} \Sigma^{T}\right) \Pi \dot{x} .
$$

Equation (12) provides a minimum Euclidean norm solution for the generalized velocities as a function of the variables $\{q, \dot{x}\}$. It is straightforward to verify that Eqs. (11) and (12) satisfy Eqs. (1) and (4). The generalized accelerations, if desired, are obtained by first solving for $\dot{\epsilon}$ by differentiating Eq. (11). $\ddot{q}$ may be determined by differentiating Eq. (12) or by applyir.g Eq. (8). The generalized coordinates are obtained by numerical integration.

In summary, given any $B(q)$ such that $\left(J^{T}(q), B^{T}(q)\right)^{T}$ is nonsingular, a value for $\epsilon$ can be determined using Eq. (11) which yields the minimum norm solution for $\dot{q}$. It should be mentioned that it is invalid to substitute $J(q) \dot{q}$ for $\dot{x}$ on the righthand side of Eq. (11) in the preceding optimization procedure because $\dot{q}$ is unknown. There would be insufficient information available to solve for $\epsilon$. Moreover, this would 
incorrectly imply that $B=-\left(\Sigma^{T} \Sigma\right)^{-1} \Sigma^{T} \Pi J$ based on the definition of $\epsilon$ in Eq. (4). Indeed, this expression for $B$ does not satisfy the matrix relations given in Eq. (5).

For redundancy resolution at the acceleration level, the redundant DOF quantified by $\dot{\epsilon}$ are determined to minimize $\|\ddot{q}\|^{2}$ using Eq. (8):

$$
\begin{aligned}
\|\ddot{q}\|^{2}= & \left\{\ddot{x}^{T} \Pi^{T}+\dot{\epsilon}^{T} \Sigma^{T}-(\Pi \dot{x}+\Sigma \epsilon)^{T}(\Pi \dot{J}+\Sigma \dot{B})^{T}\right\}\{\Pi \ddot{x}+\Sigma \dot{\epsilon} \\
& -(\Pi \dot{J}+\Sigma \dot{B})(\Pi \dot{x}+\Sigma \epsilon)\} .
\end{aligned}
$$

Taking the partial derivative of Eq. (13) with respect to $\dot{\epsilon}$, equating the result to zero, and rearranging yield a solution for $\dot{\epsilon}$ :

$$
\dot{\epsilon}=-\left(\Sigma^{T} \Sigma\right)^{-1} \Sigma^{T}[\Pi \ddot{x}-(\Pi \dot{J}+\Sigma \dot{B})(\Pi \dot{x}+\Sigma \epsilon)]
$$

In Eq. (14), the quantities $\{\Pi, \Sigma\}$ are unknown. To determine $\dot{\epsilon}$ using Eq. (14), the designer chooses matrix $B$ which immediately yields $[\Pi(q), \Sigma(q)]$ by Eq. (5). The time derivatives of $\{J, B\}$ are then obtained. Given an initial value for $\epsilon$ (at the starting time) or using its last known value (e.g., from the previous sampling period), sufficient information is now available to compute $\dot{\epsilon}$.

Substituting the right-hand side of Eq. (14) into Eq. (8), rearranging terms, and simplifying give:

$$
\ddot{q}=\left(I_{N \times N}-\Sigma\left(\Sigma^{T} \Sigma\right)^{-1} \Sigma^{T}\right) \Pi(\ddot{x}-\dot{J}(\Pi \dot{x}+\Sigma \epsilon))
$$

Equation (15) provides a minimum norm solution for the generalized accelerations as a function of the variables $\{q, \epsilon, \dot{x}, \ddot{x}\}$. It is straightforward to verify that Eqs. (14) and (15) satisfy Eqs. (2) and (6). The solution for $\epsilon$ is obtained by numerically integrating Eq. (14). The generalized coordinates and velocities are obtained by numerically integrating Eq. (15).

\subsection{SELECTION OF $B$ SUCH THAT $\epsilon$ IS A SUBSET OF $\dot{q}$}

In our first approach, we choose $B$ to relegate the control of the redundant DOF to $(N-M)$ of the $N$ joints. In this case $B$ is a constant matrix consisting of ones and zeros. The approach is illustrated by examples.

Example 1: The problem is to control the Cartesian translational and rotational motions of the end effector of the CESARm research manipulator $[6,10,11,12]$ operating in a three-dimensional workspace, as shov'n in Fig. 2. In this configuration CESARm has a single degree of redundancy with $N=7, M=6$, and $\dot{x}=\left[\left(v_{0}^{7}\right)^{T},\left(w_{0}^{7}\right)^{T}\right]^{T}$. Suppose we relegate the control of the redundant DOF to the third (upper arm roll) joint, i.e., $\epsilon=\dot{q}_{3}$. The choice is reasonable and logical. Indeed, it is the inclusion of that joint which makes CESARm redundant. $B$ is defined as: 


$$
B=[0,0,1,0,0,0,0] .
$$

Given the $(6 \times 7)$ Jacobian matrix $J(q)$ defined in Eq. (1) and the choice of vector $B$ in Eq. (16), the $(7 \times 7)$ composite matrix $\left(J^{T}(q), B^{T}\right)^{T}$ can be inverted symbolically using the method of inverse by partitioning [13]:

$$
\begin{aligned}
& \Pi=\left[\begin{array}{llllll}
1 & 0 & 0 & 0 & 0 & 0 \\
0 & 1 & 0 & 0 & 0 & 0 \\
0 & 0 & 0 & 0 & 0 & 0 \\
0 & 0 & 1 & 0 & 0 & 0 \\
0 & 0 & 0 & 1 & 0 & 0 \\
0 & 0 & 0 & 0 & 1 & 0 \\
0 & 0 & 0 & 0 & 0 & 1
\end{array}\right]\left\{\left[\begin{array}{llllll}
1 & 0 & 0 & 0 & 0 & 0 \\
0 & 1 & 0 & 0 & 0 & 0 \\
0 & 0 & 0 & 0 & 0 & 0 \\
0 & 0 & 1 & 0 & 0 & 0 \\
0 & 0 & 0 & 1 & 0 & 0 \\
0 & 0 & 0 & 0 & 1 & 0 \\
0 & 0 & 0 & 0 & 0 & 1
\end{array}\right]\right\}^{-1}, \\
& \Sigma\left.\left.=\left(\begin{array}{llllll}
1 & 0 & 0 & 0 & 0 & 0 \\
0 & 1 & 0 & 0 & 0 & 0 \\
0 & 0 & 0 & 0 & 0 & 0 \\
0 & 0 & 1 & 0 & 0 & 0 \\
0 & 0 & 0 & 1 & 0 & 0 \\
0 & 0 & 0 & 0 & 1 & 0 \\
0 & 0 & 0 & 0 & 0 & 1
\end{array}\right]\left\{\left[\begin{array}{llllll}
1 & 0 & 0 & 0 & 0 & 0 \\
0 & 1 & 0 & 0 & 0 & 0 \\
0 & 0 & 0 & 0 & 0 & 0 \\
0 & 0 & 1 & 0 & 0 & 0 \\
0 & 0 & 0 & 1 & 0 & 0 \\
0 & 0 & 0 & 0 & 1 & 0 \\
0 & 0 & 0 & 0 & 0 & 1
\end{array}\right]\right\}^{-1} J\right) B^{T}\right) \\
&=\left(I_{7 \times 7}-\Pi J\right) B^{T}
\end{aligned}
$$

where $\Pi$ is a $(7 \times 6)$ matrix, $\Sigma$ is a $(7 \times 1)$ vector and, here again, $I_{r \times r}$ denotes an $(r \times r)$ identity matrix. Upon calculating $\Pi(q)$ and $\Sigma(q), \epsilon$ and $\dot{q}$ are obtained usings Eqs. (11) and (12), respectively, when it is desired to minimize $\|\dot{q}\|^{2}$.

Example 2: The problem is to control the Cartesian translational motion of the end effector of the CESARm research manipulator at its centerpoint. In this configuration CESARm is a spatial mechanism with four degrees of redundancy $\left(N=7, M=3, \dot{x}=v_{0}^{7}\right)$. A practical application of such a configuration is having the end effector trace a circle where its orientation is of no consequence. We choose $\epsilon=\left[\dot{q}_{3}, \dot{q}_{5}, \dot{q}_{6}, \dot{q}_{7}\right]^{T}$, thus:

$$
B=\left[\begin{array}{lllllll}
0 & 0 & 1 & 0 & 0 & 0 & 0 \\
0 & 0 & 0 & 0 & 1 & 0 & 0 \\
0 & 0 & 0 & 0 & 0 & 1 & 0 \\
0 & 0 & 0 & 0 & 0 & 0 & 1
\end{array}\right]
$$




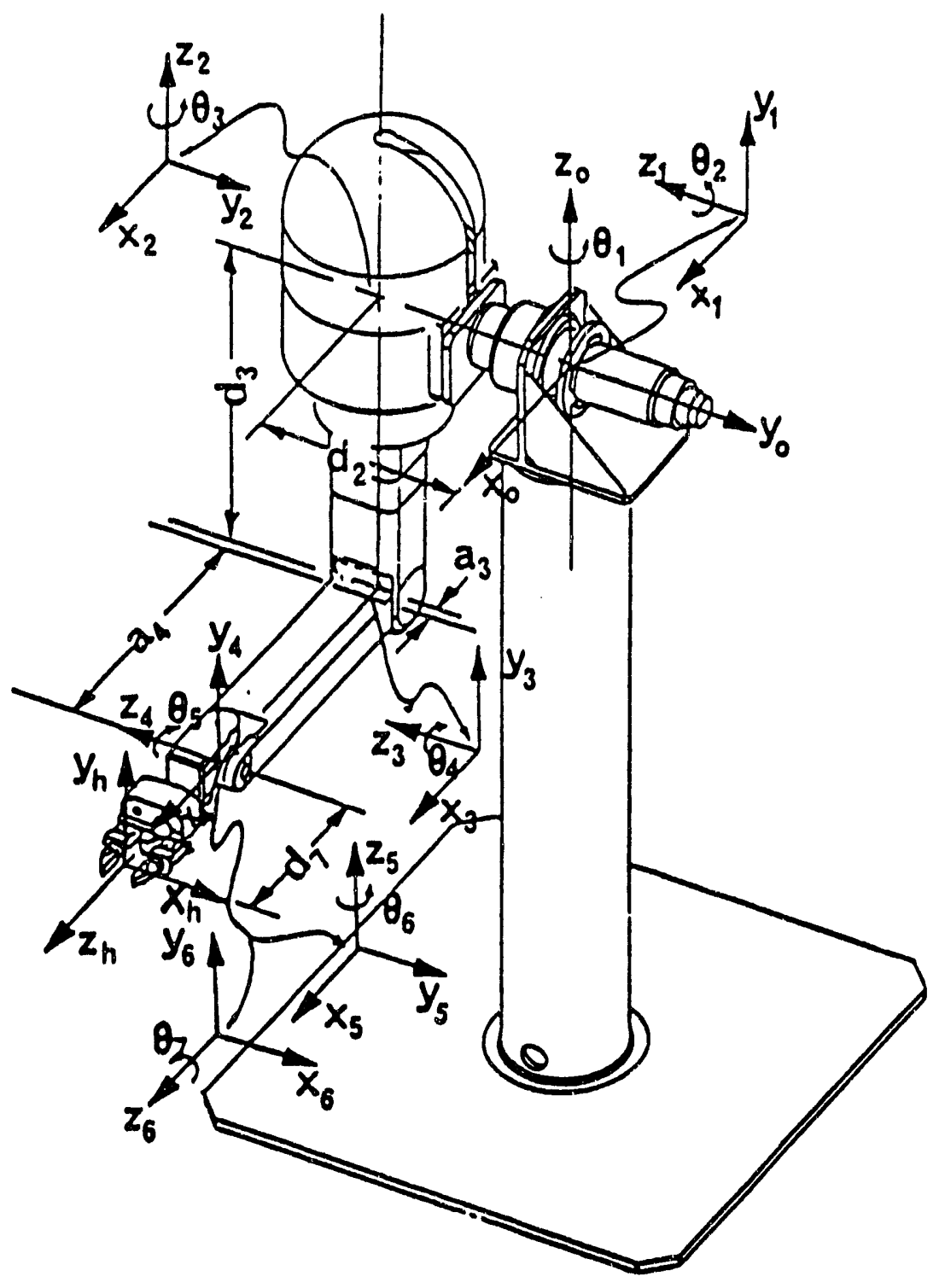

Fig. 2. System configuration and coordinate system assignment for the CESA.Rm research manipulator. 
Given the $(3 \times 7)$ matrix $J(q)$ defined in Eq. (1) and matrix $B$ defined by Eq. (19), the $(7 \times 7)$ matrix $\left(J^{T}(q), B^{T}\right)^{T}$ can be inverted symbolically [13]:

$$
\begin{aligned}
& \Pi=\left[\begin{array}{lll}
1 & 0 & 0 \\
0 & 1 & 0 \\
0 & 0 & 0 \\
0 & 0 & 1 \\
0 & 0 & 0 \\
0 & 0 & 0 \\
0 & 0 & 0
\end{array}\right]\left\{J\left[\begin{array}{lll}
1 & 0 & 0 \\
0 & 1 & 0 \\
0 & 0 & 0 \\
0 & 0 & 1 \\
0 & 0 & 0 \\
0 & 0 & 0 \\
0 & 0 & 0
\end{array}\right]\right\}^{-1}, \\
& \Sigma\left.=\left(I_{7 \times 7}-\left[\begin{array}{lll}
1 & 0 & 0 \\
0 & 1 & 0 \\
0 & 0 & 0 \\
0 & 0 & 1 \\
0 & 0 & 0 \\
0 & 0 & 0 \\
0 & 0 & 0
\end{array}\right]\left\{\left[\begin{array}{lll}
1 & 0 & 0 \\
0 & 1 & 0 \\
0 & 0 & 0 \\
0 & 0 & 1 \\
0 & 0 & 0 \\
0 & 0 & 0 \\
0 & 0 & 0
\end{array}\right]\right\}^{-1} J\right) B^{T}\right) \\
&=\left(I_{7 \times 7}-\Pi J\right) B^{T}
\end{aligned}
$$

where $\Pi$ and $\Sigma$ are $(7 \times 3)$ and $(7 \times 4)$ matrices, respectively.

The principal benefit of choosing $B$ in this way is that the inversion of the $(N \times N)$ matrix $\left(J^{T}(q), B^{T}\right)^{T}$ is reduced to inverting the $(M \times M)$ matrix consisting of $M$ columns of the Jacobian $J$. Moreover, by choosing $B$ as a constant matrix, the problem of deriving an analytical expression for $\dot{B}$ is avoided.

Interestingly, the particular $\dot{q}_{p}$ and homogeneous $\dot{q}_{h}$ solutions to Eq. (1) proposed in [6] are just special cases of the components $\{\Pi \dot{x}\}$ and $\{\Sigma \epsilon\}$ of the general solution for $\dot{q}$ proposed in Eq. (7). Indeed, by selecting $B$ as a constant matrix such that $\epsilon$ is a subset of $\dot{q}$, the results of [6] have been obtained here as shown in Example 1 for manipulators with one degree of redundancy. For the case of multiple degrees of redundancy, a procedure is suggested in [6] which is equivalent to transforming the $(N-M)$ linearly independent columns of the orthogonal complement $\Sigma$ so they become mutually orthogonal using the Gram-Schmidt procedure [14]. Such techniques are numerically intensive and may produce computed vectors that are far from orthogonal [14]. The case of picking $B$ such that $\epsilon$ is a subset of the generalized velocities as presented here is motivated in part by its simplicity. The columns of $\Sigma$ obtained by the method are only linearly independent. The benefits of orthogonalizing the columns of $\Sigma$ as suggested in [6] are not clearly demonstrated. Besides, this would require recalculating $B$ to ensure that Eq. (5) is satisfied in the method presented here. Thus the minimum Euclidean norm solution for $\dot{q}$ given in Eq. (12) differs from that in [6] when $N-M>1$. The problem of resolving the kinematic redundancy at the acceleration level is not addressed in [6]. 


\subsection{CHOOSING $B$ ORTHOGONAL TO THE ROWS OF $J$}

This approach to selecting $B(q)$ is inspired in part by the recent work on the modeling and force control of the constrained nonholonomic motion of a platform with multiple, steerable wheels [15]. The partitioned inverse in Eq. (5) only requires that $J$ and $B$ be full rank matrices, and further that the rows of $B$ be linearly independent of the rows of $J$. Here a method is suggested for choosing $B(q)$ to be orthogonal to the rows of $J(q)$. It is assumed that the serial-link manipulator has a single redundant $\operatorname{DOF}(N=M+1)$, e.g., the CESARm research manipulator in its most general configuration with $M=6$ and $N=7[6,10,11,12]$. Thus $\epsilon$ is a scalar and $B(q)$ is an $N$-dimensional row vector.

Expanding along the last row of $\left(J^{T}(q), B^{T}(q)\right)^{T}$ using the Laplace expansion [13], the determinant of $\left(J^{T}(q), B^{T}(q)\right)^{T}$ is:

$$
\operatorname{det}\left[\begin{array}{l}
J(q) \\
B(q)
\end{array}\right]=B(q) \Delta
$$

where $\operatorname{det}[]$ denotes the determinant of []. In Eq. (22), $\Delta\left[=\left(\Delta_{N 1}, \Delta_{N 2}, \ldots, \Delta_{N N}\right)^{T}\right]$ is the vector of cofactors, where $\Delta_{N i}$ is the cofactor of the $i$ th element of the $N$ th row of $\left(J^{T}(q), B^{T}(q)\right)^{T}$. Vector $\Delta$ is a function of the generalized coordinates, but not of the elements of $B$.

The general class of $(N \times 1)$ orthogonal complements $\Sigma$ of $J$ is defined as the last column of $\left\{\left(J^{T}(q), B^{T}(q)\right)^{T}\right\}^{-1}$ :

$$
\Sigma=\frac{1}{\operatorname{det}\left[\begin{array}{l}
J(q) \\
B(q)
\end{array}\right]} \Delta
$$

where a specific $\Sigma$ is obtained by the designer's choice of $B$.

We select $B^{T}$ to maximize the determinant of $\left[J^{T}(q), B^{T}(q)\right]^{T}$ subject to the constraint of normalizing the Euclidean norm of $B^{T}$ to a constant value, i.e., $\left\|B^{T}\right\|^{2}=\mu$, where the positive scalar $\mu$ is a normalization factor selected by the designer. We introduce the Lagrangian $\mathcal{L}$ :

$$
\mathcal{L}\left(B^{T}, \lambda\right)=\Delta^{T} B^{T}+\lambda\left(B B^{T}-\mu\right)
$$

in which Eq. (22) has been invoked. Taking the partial derivative of $\mathcal{L}$ with respect to the vector $B^{T}$ and scalar $\lambda$, respectively, and equating the results to zero provide the necessary optimality conditions:

$$
\begin{gathered}
\Delta+2 \lambda B^{T}=0_{N \times 1}, \\
B B^{T}-\mu=0 .
\end{gathered}
$$


14 RESOLUTION OF KINEMATIC REDUNDANCY

Equation (25) can be solved for $B^{T}$ :

$$
B^{T}=-\frac{1}{2 \lambda} \Delta
$$
Eliminating $\left\{B, B^{T}\right\}$ from Eq. (26) using the right-hand side of Eq. (27) and
rearranging terms give:

$$
\lambda^{2}=\frac{1}{4 \mu} \Delta^{T} \Delta
$$

which yields a solution for $\lambda$ :

$$
\lambda=-\frac{1}{2} \sqrt{\frac{\Delta^{T} \Delta}{\mu}}
$$

where the negative sign in the general solution $\pm \frac{1}{2} \sqrt{\left(\Delta^{T} \Delta\right) / \mu}$ for $\lambda$ has been selected to maximize $\operatorname{det}\left[J^{T}(q), B^{T}(q)\right]^{T}$. Indeed, it is easy to see that $\partial^{2} \mathcal{L} / \partial B^{T^{2}}\left(=2 \lambda I_{N \times N}\right)$ is negative semidefinite when $\lambda$ is defined by Eq. (29). $B$ :

Backsubstituting Eq. (29) into Eq. (27) and transposing yield the solution for

$$
B=\frac{\sqrt{\mu} \Delta^{T}}{\sqrt{\Delta^{T} \Delta}}
$$

Substituting the right-hand side of Eq. (30) into Eq. (22) reveals that:

$$
\operatorname{det}\left[\begin{array}{c}
J(q) \\
B(q)
\end{array}\right]=\sqrt{\Delta^{T} \Delta \mu}
$$

A relationship between $B$ and $\Sigma$ can be obtained from Eqs. (23) and (31):

$$
\Sigma=\frac{B^{T}}{\mu} \text {. }
$$

Since $J \Sigma=0_{M \times 1}$, then $B$ is orthogonal to the rows of $J$. Furthermore, postmultiplying the matrix identity $\Pi J+\Sigma B=I_{N \times N}$ by $J^{T}$ gives:

$$
\Pi J J^{T}=J^{T} .
$$

Since $J$ has full rank $M$, then matrix $\left(J J^{T}\right)$ is symmetric, positive definite, and therefore nonsingular. Thus Eq. (33) can be solved for the right inverse $\Pi$ of $J$ :

$$
\Pi=J^{T}\left(J J^{T}\right)^{-1} \text {. }
$$


It is immediately evident that the columns of $\Pi$ are orthogonal to the columns of $\Sigma$ :

$$
\Pi^{T} \Sigma=0_{M \times 1} .
$$

The general solution for $\dot{q}$ given in Eq. (7) becomes:

$$
\dot{q}=J^{T}\left(J J^{T}\right)^{-1} \dot{x}+\frac{\Delta}{\sqrt{\Delta^{T} \Delta \mu}} \epsilon .
$$

When the redundant DOF are relegated to achieve a minimum Euclidean norm solution for $\dot{q}$, we substitute Eq. (35) into Eq. (11) to yield $\epsilon=0$. Thus Eq. (36) simplifies to $\dot{q}=J^{T}\left(J J^{T}\right)^{-1} \dot{x}$ which is the classic Moore Penrose pseudoinverse solution for the generalized velocities [2,3]. Furthermore, the minimum Euclidean norm solution for the generalized accelerations given by Eq. (15) simplifies to:

$$
\ddot{q}=J^{T}\left(J J^{T}\right)^{-1}\left(\ddot{x}-j\left(J^{T}\left(J J^{T}\right)^{-1} \dot{x}+\frac{\Delta}{\sqrt{\Delta^{T} \Delta \mu}} \epsilon\right)\right) .
$$

Example 3: The problem is to control the Cartesian translational motion of the centerpoint of the wrist of the CESARm research manipulator as shown in Fig. 2. In this configuration CESARm has a single redundant DOF with $M=3, N=4$, and $\dot{x}=v_{0}^{4}$. The matrix $\left(J^{T}, B^{T}\right)^{T}$ can be expanded to reveal its component elements:

$$
\left[\begin{array}{l}
J \\
B
\end{array}\right]=\left[\begin{array}{llll}
j_{11} & j_{12} & j_{13} & j_{14} \\
j_{21} & j_{22} & j_{23} & j_{24} \\
j_{31} & j_{32} & j_{33} & j_{34} \\
b_{11} & b_{12} & b_{13} & b_{14}
\end{array}\right]
$$

Expanding along the fourth row of matrix $\left(J^{T}, B^{T}\right)^{T}$ defined in Eq. (38), the vector of cofactors $\Delta\left(=\left[\Delta_{41}, \Delta_{42}, \Delta_{43}, \Delta_{44}\right]^{T}\right)$ is given by:

$$
\Delta=\left[\begin{array}{c}
-j_{12}\left(j_{23} j_{34}-j_{24} j_{33}\right)+j_{13}\left(j_{22} j_{34}-j_{24} j_{32}\right)-j_{14}\left(j_{22} j_{33}-j_{23} j_{32}\right) \\
j_{11}\left(j_{23} j_{34}-j_{24} j_{33}\right)+j_{13}\left(j_{24} j_{31}-j_{21} j_{34}\right)+j_{14}\left(j_{21} j_{33}-j_{23} j_{31}\right) \\
-j_{11}\left(j_{22} j_{34}-j_{24} j_{32}\right)+j_{12}\left(j_{21} j_{34}-j_{24} j_{31}\right)-j_{14}\left(j_{21} j_{32}-j_{22} j_{31}\right) \\
j_{11}\left(j_{22} j_{33}-j_{23} j_{32}\right)+j_{12}\left(j_{23} j_{31}-j_{21} j_{33}\right)+j_{13}\left(j_{21} j_{32}-j_{22} j_{31}\right)
\end{array}\right]
$$

The Jacobian matrix for this particular configuration of CESARm is defined in Appendix A. Substituting for $j_{l m}$ in Eq. (39) using the appropriate equation from Appendix A yields the solution for the cofactors $\Delta_{4 i}(i=1,2,3,4)$ as a function of the lower four joint angles of CESARm:

$$
\begin{aligned}
\Delta_{41}= & -\left\{a_{3} a_{4}^{2} c_{3} c_{4} s_{4}+a_{3}^{2} a_{4} c_{3} s_{4}+a_{4}^{2} d_{3} c_{3} c_{4}^{2}\right. \\
& \left.+a_{3} a_{4} d_{3} c_{3} c_{4}\right\}
\end{aligned}
$$




$$
\begin{aligned}
\Delta_{42}= & a_{3} a_{4}^{2} s_{2} s_{3} c_{4} s_{4}-a_{4}^{2} d_{2} s_{2} c_{4} s_{4}+a_{3}^{2} a_{4} s_{2} s_{3} s_{4} \\
& -a_{3} a_{4} d_{2} s_{2} s_{4}+a_{4}^{2} d_{3} s_{2} s_{3} c_{4}^{2}+a_{4}^{2} d_{2} c_{2} c_{3} c_{4}^{2} \\
& +a_{3} a_{4} d_{3} s_{2} s_{3} c_{4}+a_{3} a_{4} d_{2} c_{2} c_{3} c_{4} \\
\Delta_{43}= & -\left\{a_{4}^{2} d_{2} c_{2} c_{3} s_{3} c_{4} s_{4}-a_{3} a_{4}^{2} c_{2} c_{3} c_{4} s_{4}+a_{4}^{2} d_{3} s_{2} c_{4} s_{4}\right. \\
+ & a_{3} a_{4} d_{2} c_{2} c_{3} s_{3} s_{4}+a_{4} d_{2} d_{3} s_{2} s_{3} s_{4}-a_{3}^{2} a_{4} c_{2} c_{3} s_{4} \\
- & a_{3} a_{4} d_{3} s_{2} s_{4}+a_{4}^{2} d_{2} s_{2} s_{3} c_{4}^{2}-a_{4}^{2} d_{3} c_{2} c_{3} c_{4}^{2} \\
- & a_{3} a_{4}^{2} s_{2} c_{4}^{2}-a_{3} a_{4} d_{3} c_{2} c_{3} c_{4}-a_{4} d_{3}^{2} s_{2} c_{4} \\
- & \left.a_{4}^{2} d_{2} s_{2} s_{3}+a_{3} a_{4}^{2} s_{2}\right\}, \\
\Delta_{44}= & a_{4}^{2} d_{2} s_{2} c_{3} c_{4} s_{4}+a_{3} a_{4} d_{2} s_{2} c_{3} s_{4}-a_{4}^{2} d_{2} c_{2} c_{3}^{2} c_{4}^{2} \\
& -2 a_{3} a_{4} d_{2} c_{2} c_{3}^{2} c_{4}-a_{4} d_{2} d_{3} s_{2} c_{3} c_{4}-a_{3}^{2} d_{2} c_{2} c_{3}^{2} \\
& -a_{3} d_{2} d_{3} s_{2} c_{3}
\end{aligned}
$$

where $c_{i}=\cos \left(q_{i}\right), s_{i}=\sin \left(q_{i}\right)$ and where $\left\{a_{3}, a_{4}, d_{2}, d_{3}\right\}$ are constant Denavit-Hartenberg link length parameters defined in Appendix $\mathrm{A}$.

In [4], the orthogonal complement was selected to be $\Sigma=\Delta$ for $M$ even and $\Sigma=-\Delta$ for $M$ odd when $N-M=1$. Although not discussed, this implies that $\operatorname{det}\left[J^{T}, B^{T}\right]^{T}= \pm 1$. It is not clear why the sign of the determinant is dependent on $M$ being even or odd. The approach presented here selects $B$ so as to maximize $\operatorname{det}\left[J^{T}, B^{T}\right]^{T}$ subject to the constraint $\left\|B^{T}\right\|^{2}=\mu$, in addition to satisfying $B J^{T}=0_{1 \times M}$. In Appendix B symbolic expressions for $\operatorname{det}\left[J^{T}, B^{T}\right]^{T}$ and $\Sigma$ are derived as functions of $B$ for the case $N-M=2$. They are the two-redundant-DOF counterparts to Eqs. (22) and (23). Additionally, a method for choosing $B$ to set $\operatorname{det}\left[J^{T}, B^{T}\right]^{T}$ to a desired reference value is discussed.

\subsection{SELECTING $B$ BY APPLICATION OF THE ZERO- EIGENVALUE MATRIX THEOREM}

An approach to selecting $B(q)$ is now suggested which is ap application of the zero-eigenvalue matrix theorem $[14,16,17,18]$. Here we only assume that $(N>M)$. With this approach, $B(q)$ will again be chosen to be orthogonal to the rows of $J(q)$.

Let us first consider the properties of the $(N \times N)$ matrix $\left(J^{T} J\right)$. It is proven in Appendix $\mathrm{C}$ by analytical techniques that $\left(J^{T} J\right)$ is a singular matrix and that its rank is equal to $M$. Furthermore, it is shown that $(N-M)$ of the eigenvalues of $\left(J^{T} J\right)$ are identically zero. Let the $(N \times 1)$ vector $Y_{i}(i=1,2, \ldots, N-M)$ denote the $i$ th eigenvector of $\left(J^{T} J\right)$ corresponding to the $i$ th zero eigenvalue. Then $\alpha Y_{i}$, where $\alpha$ is any nonzero scalar, is also an eigenvector of $\left(J^{T} J\right)$ associated with the $i$ th zero eigenvalue. This indeterminancy can be removed by further requiring that $\left\|Y_{i}\right\|^{2}=1$. It is straightforward to verify that: 


$$
J^{T} J\left[Y_{1}, Y_{2}, \cdots, Y_{N-M}\right]=0_{(N \times(N-M))} .
$$

Premultiplying Eq. (44) by $\Pi^{T}$ yields:

$$
J\left[Y_{1}, Y_{2}, \cdots, Y_{N-M}\right]=0_{(M \times(N-M))}
$$

in which Eq. (5) has been invoked. Equation (45) reveals an obvious choice for matrix $B$ whose rows are orthogonal to those of $J$ :

$$
B=\left[\begin{array}{c}
Y_{1}^{T} \\
Y_{2}^{T} \\
\vdots \\
Y_{N-M}^{T}
\end{array}\right]
$$

Given matrix $B$ as defined, in Eq. (46), suppose matrix $\Sigma$ is chosen as:

$$
\Sigma=B^{T}\left(B B^{T}\right)^{-1}
$$

where for computational reasons it should be mentioned that the main diagonal elements of $\left(B B^{T}\right)$ are all ones. It is easy to see that $J \Sigma=0_{(M \times(N-M))}$ and $\Pi^{T} \Sigma=0_{(M \times(N-M))}$, where matrix $\Pi$ is defined by Eq. (34).

When picking vector $\epsilon$ to minimize $\|\dot{q}\|^{2}$ as shown in Eqs. (9) and (11), it is easy to see that $\epsilon=0_{(N-M) \times 1}$ and $\dot{q}=J^{T}\left(J J^{T}\right)^{-1} \dot{x}$ when $B$ is defined by Eq. (46). The minimum Euclidean norm solution for the generalized accelerations can be obtained by substituting the above results into Eq. (15). Since analytical methods for obtaining $B$ such that $J B^{T}=0_{(M \times(N-M))}$ have been developed in the preceding section for $N-M=1$, the zero-eigenvalue matrix method is particularly useful for the case of $N-M \geq 2$. It is a numerical approach that requires determining the eigenvectors $Y_{i}$ using various computational iterative algorithms $[18,19]$.

\subsection{THE VECTOR CROSS PRODUCT METHOD FOR PLANAR MANIPULATORS}

In this section a method is proposed for determining a full rank orthogonal complement of the Jacobian matrix for the inverse kinematics of a serial-link planar manipulator with revolute joints. The planar manipulator shown in Fig. 3 contains $N$ revolute joints, where $N>2$. Only the translational motion of the end effector is to be specified and controlled. Therefore, the planar system contains $(N-2)$ redundant DOF. Let the $(2 \times 1)$ vectors $p_{0}^{N}\left(=\left[p_{0 x}^{N}, p_{0 y}^{N}\right]^{T}\right)$ and $v_{0}^{N}\left(=\left[v_{0 x}^{N}, v_{0 y}^{N}\right]^{T}\right)$ denote the Cartesian translational position and velocity of the end effector in the base coordinate system. $p_{0}^{N}$ is related to the joint positions as follows: 


$$
p_{0}^{N}=\left[\begin{array}{l}
l_{1} c\left(q_{1}\right)+l_{2} c\left(q_{1}+q_{2}\right)+\ldots+l_{N} c\left(q_{1}+q_{2}+\ldots+q_{N}\right) \\
l_{1} s\left(q_{1}\right)+l_{2} s\left(q_{1}+q_{2}\right)+\ldots+l_{N} s\left(q_{1}+q_{2}+\ldots+q_{N}\right)
\end{array}\right]
$$

where $l_{i}$ is the constant length of link $i$ and $c(\cdot)=\cos (\cdot), s(\cdot)=\sin (\cdot)$.

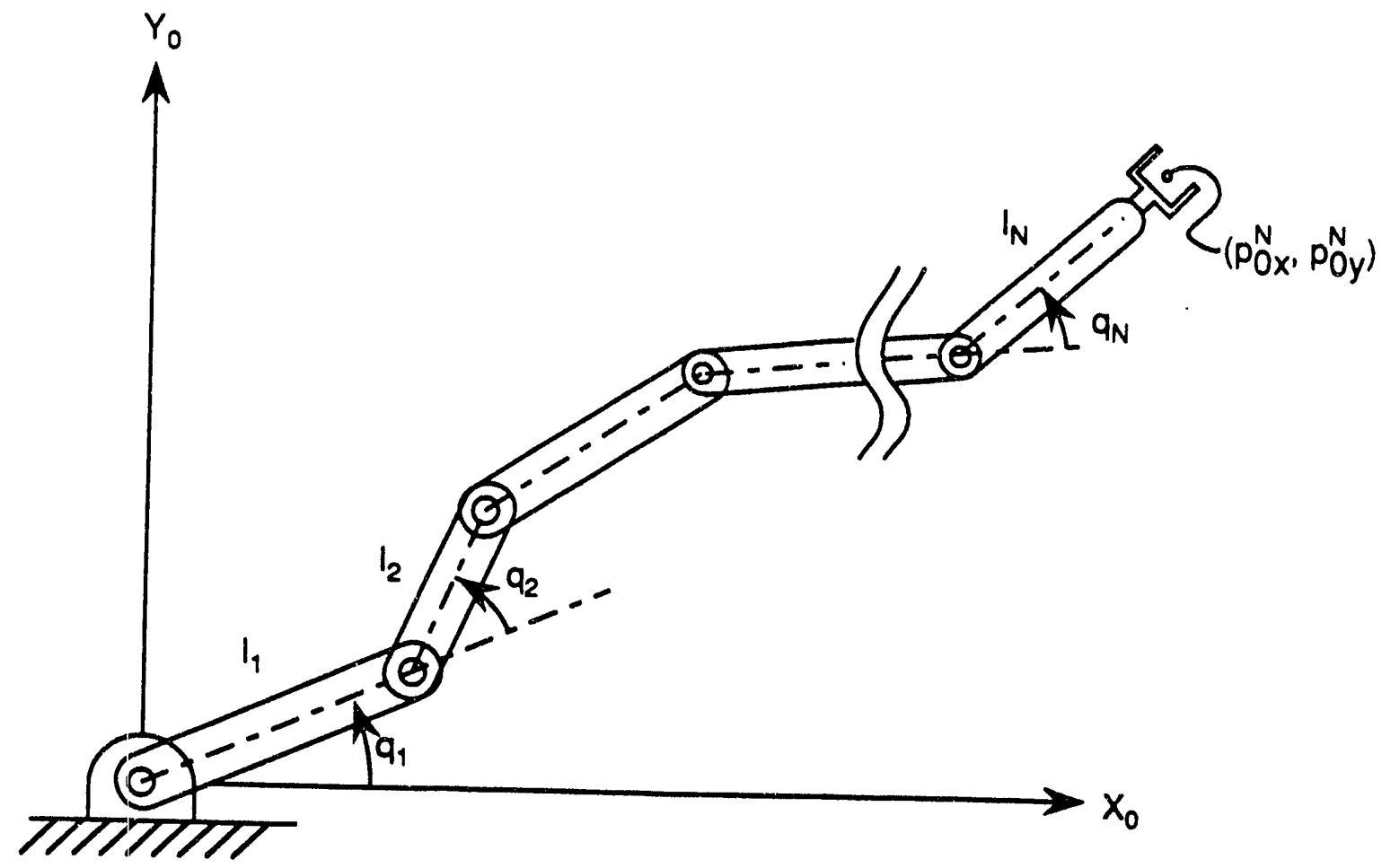

Fig. 3. Planar revolute redundant manipulator.

Differentiating Eq. (48) provides a relation between the joint velocities and the Cartesian translational velocities of the end effector:

$$
v_{0}^{N}=J(q) \dot{q}
$$

where $q=\left[q_{1}, q_{2}, \ldots, q_{N}\right]^{T}$. In Eq. (49), the $(2 \times N)$ Jacobian matrix $J(q)$ is
defined by:

$$
J=\left[\begin{array}{lllll}
j_{11}, & j_{12}, & j_{13}, & \ldots, & j_{1 N} \\
j_{21}, & j_{22}, & j_{23}, & \ldots, & j_{2 N}
\end{array}\right]
$$

where its elements are defined by: 


$$
\begin{gathered}
j_{11}=-l_{1} s\left(q_{1}\right)-l_{2} s\left(q_{1}+q_{2}\right)-\ldots-l_{N} s\left(q_{1}+q_{2}+\ldots+q_{N}\right) \\
j_{12}=-l_{2} s\left(q_{1}+q_{2}\right)-\ldots-l_{N} s\left(q_{1}+q_{2}+\ldots+q_{N}\right) \\
\vdots \\
j_{1 N}=-l_{N} s\left(q_{1}+q_{2}+\ldots+q_{N}\right) \\
j_{21}=l_{1} c\left(q_{1}\right)+l_{2} c\left(q_{1}+q_{2}\right)+\ldots+l_{N} c\left(q_{1}+q_{2}+\ldots+q_{N}\right) \\
j_{22}=l_{2} c\left(q_{1}+q_{2}\right)+\ldots+l_{N} c\left(q_{1}+q_{2}+\ldots+q_{N}\right) \\
\vdots \\
j_{2 N}=l_{N} c\left(q_{1}+q_{2}+\ldots+q_{N}\right)
\end{gathered}
$$

The problem is to determine the $(N \times(N-2))$ orthogonal complement $\Sigma$ of the matrix $J$ such that $J \Sigma=0_{(2 \times(N-2)) \text {. We propose the "vector cross product }}$ method" to obtain $\Sigma$. The $(k-2)$ th column of $\Sigma$ is determined by taking the vector cross product of two $(3 \times 1)$ vectors $\left\{v_{1 k}, v_{2 k}\right\}$ whose components consist of subsets of the rows of the Jacobian. $v_{1 k}$ and $v_{2 k}$ are defined by:

$$
\begin{gathered}
v_{1 k}=\left[j_{11}, j_{12}, j_{1 k}\right]^{T}, \\
v_{2 k}=\left[j_{21}, j_{22}, j_{2 k}\right]^{T}
\end{gathered}
$$

where $k=3,4, \ldots, N$.

The cross product between $v_{1 k}$ and $v_{2 k}$ may be expressed in a matrix-column vector notation $[13]$ :

$$
v_{r s l t k}=\left[\begin{array}{ccc}
0, & -j_{1 k}, & j_{12} \\
j_{1 k}, & 0, & -j_{11} \\
-j_{12}, & j_{11}, & 0
\end{array}\right]\left[\begin{array}{l}
j_{21} \\
j_{22} \\
j_{2 k}
\end{array}\right]=\left[\begin{array}{l}
j_{12} j_{2 k}-j_{1 k} j_{22} \\
j_{1 k} j_{21}-j_{11} j_{2 k} \\
j_{11} j_{22}-j_{12} j_{21}
\end{array}\right]
$$

where $(3 \times 1)$ vector $v_{\text {ralt }}$ is the resultant of the cross product. 
Let $(N \times 1)$ vector $\Sigma_{(k-2)}$ denote the $(k-2)$ th column of $\Sigma(k=3,4, \ldots, N)$. It is defined by:

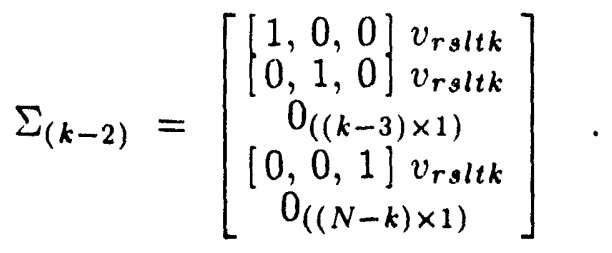

By invoking the definition of $v_{\text {rsltk }}$ in Eq. (59), it is seen that $J \Sigma_{(k-2)}=0_{2 \times 1}$.

Example 4: Suppose $N=5$. The orthogonal complement of the $(2 \times 5)$ Jacobian $J$ is given by:

$$
\Sigma=\left[\begin{array}{ccc}
j_{12} j_{23}-j_{13} j_{22} & j_{12} j_{24}-j_{14} j_{22} & j_{12} j_{25}-j_{15} j_{22} \\
j_{13} j_{21}-j_{11} j_{23} & j_{14} j_{21}-j_{11} j_{24} & j_{15} j_{21}-j_{11} j_{25} \\
j_{11} j_{22}-j_{12} j_{21} & 0 & 0 \\
0 & j_{11} j_{22}-j_{12} j_{21} & 0 \\
0 & 0 & j_{11} j_{22}-j_{12} j_{21}
\end{array}\right]
$$

in which Eqs. (59) and (60) have been applied. Matrix $\Sigma$ can be expressed as a function of the five joint angles $\left\{q_{1}, q_{2}, \ldots, q_{5}\right\}$ by applying Eqs. (51)-(56) with $N=5$.

Given $\Sigma=\left[\Sigma_{1}, \Sigma_{2}, \ldots, \Sigma_{(N-2)}\right]$, suppose we choose the $((N-2) \times N)$ matrix $B(q)$ such that:

$$
B=\left(\Sigma^{T} \Sigma\right)^{-1} \Sigma^{T}
$$

Then it is easy to verify that $J B^{T}=0_{(2 \times(N-2))}$ and that $\Pi$ is defined by Eq. (34).

In [4], a set of equations of the form of Eq. (4) was introduced with the restriction that $\epsilon=0_{((N-M) \times 1)}$. Equations (1) and (4) were combined and solved for $\dot{q}$. The square matrix $\left(J^{T}, B^{T}\right)^{T}$ was referred to as the extended Jacobian. Matrix $B$ was obtained by taking the Lie derivative of a scalar objective function to be optimized in the independent directions of the null space of $J$ to yield $J B^{T}=0_{(M \times(N-M))}$. The problem of determining the null space of the Jacobian, that is, the orthogonal complement $\Sigma$, is not addressed for the case of $N-M>1$ in [4]. In the approach used here, the components of $\epsilon$ can be nonzero. The general development leading to Eqs. (7) and (8) only requires that the rows of $B$ be linearly independent of the rows of $J$. As a special case it is shown that $B$ can be selected to be orthogonal to the rows of $J$, which results in $\epsilon=0_{((N-M) \times 1)}$ when minimizing the Euclidean norm $\|\dot{q}\|^{2}$. In Section 3.2 it has been shown that matrices $\Pi$ and $\Sigma$ can be determined analytically thus avoiding the numerical inversion of $\left(J^{T}, B^{T}\right)^{T}$ for calculating their values, which was not addressed in [4].

The general solution for $\ddot{q}$ given by Eq. (8) obtained when resolving the kinematic redundancy at the acceleration level is useful in the development of 
a dynamical model and control architecture for the gross motion of a redundant manipulator. This is discussed next. 


\section{RIGID BODY DYNAMICAL MODEL AND CONTROL ARCHITECTURE}

A dynamical model for the entire system is formed by first combining the joint space dynamical model of the manipulator with the inverse kinematic solutions derived in the previous section. The model is then transformed to separate it into two distinct sets of equations of motion. Additionally, a control architecture of the input relegation decoupling type [1] is suggested based on the model.

To determine a rigid body model, the generalized velocities and accelerations are eliminated from the joint space dynamical model in Eq. (3) using Eqs. (7) and (8):

$$
D\{\Pi \ddot{x}+\Sigma \dot{\epsilon}\}=\tau+D\{\Pi \dot{J}+\Sigma \dot{B}\}\{\Pi \dot{x}+\Sigma \epsilon\}-C-G
$$

Premultiplying Eq. (63) by the $(N \times N)$ nonsingular matrix $\left[D^{-1}\left[J^{T}, B^{T}\right]\right]^{T}$ and utilizing the matrix relations in Eq. (5) separates the model into two sets of equations:

$$
\begin{gathered}
\ddot{x}=J D^{-1}\{\tau-C-G\}+\dot{J}\{\Pi \dot{x}+\Sigma \epsilon\}, \\
\dot{\epsilon}=B D^{-1}\{\tau-C-G\}+\dot{B}\{\Pi \dot{x}+\Sigma \epsilon\}
\end{gathered}
$$

The $M$ second order differential equations of motion governing the motion of the point, link, or end effector being controlled in Cartesian space are given by Eq. (64) as functions of the variables $\{\dot{x}, \ddot{x}, \epsilon, q, \tau\}$. On the other hand, Eq. (65) is comprised of $(N-M)$ second order differential equations of motion which govern the behavior of the redundant DOF in terms of the variables $\{\dot{x}, \epsilon, \dot{\epsilon}, q, \tau\}$.

The problem now considered is to determine a control input $\tau$ to Eqs. (64) and (65) so that the Cartesian variables $\{\dot{x}, \ddot{x}\}$ and the redundant DOF variables $\{\epsilon, \dot{\epsilon}\}$ will be controlled independently. The proposed controller consists of the sum of the outputs of a $(N \times 1)$ primary controller $\left(\tau^{p}\right)$ that is designed for cancellation of nonlinear terms in the model and a $(N \times 1)$ secondary controller $\left(\tau^{s}\right)$ that performs closed-loop servoing. The composite control $(\tau)$ is specified as $\tau=\tau^{p}+\tau^{s}$. The primary and secondary controllers are defined by:

$$
\begin{gathered}
\tau^{p}=-\hat{D}\{\hat{\Pi} \hat{j}+\hat{\Sigma} \hat{\dot{B}}\}\{\hat{\Pi} \hat{\dot{x}}+\hat{\Sigma} \hat{\epsilon}\}+\hat{C}+\hat{G}, \\
\tau^{s}=\hat{D}\left[\hat{\Pi} \tau_{\text {cart }}^{s}+\hat{\Sigma} \tau_{\text {redun }}^{s}\right]
\end{gathered}
$$

where the superscript denotes that the quantity is estimated as a function of the desired (reference) feedforward trajectory $\left(q^{r e f}, \epsilon^{r e f}, \dot{x}^{r e f}\right)$ and/or the feedback 
variables $(q, \epsilon, \dot{x})$. In Eq. $(67), \tau_{\text {cart }}^{s}$ and $\tau_{\text {redun }}^{s}$ are $(M \times 1)$ and $((N-M) \times 1)$ vectors, respectively, representing new control input variables to be determined.

(67) into Eqs. (64) and (65), under the assumption that the follow by Eq. (66) and

$$
\begin{gathered}
\hat{D}=D \quad \hat{C}=C \quad \hat{G}=G \quad \hat{J}=J \quad \hat{B}=B \quad \hat{j}=\dot{J}, \\
\hat{\dot{B}}=\dot{B} \quad[\hat{\Pi}, \hat{\Sigma}]=\left\{\left[J^{T}, B^{T}\right]^{T}\right\}^{-1}
\end{gathered}
$$

leads to the closed-loop system:

$$
\begin{aligned}
& \ddot{x}=\tau_{\text {cart }}^{s}, \\
& \dot{\epsilon}=\tau_{\text {redun }}^{s}
\end{aligned}
$$

in which Eq. (5) has been used. Suppose $\tau_{\text {cart }}^{s}$ and $\tau_{\text {redun }}^{s}$ are selected by the designer to control $\{\dot{x}, \ddot{x}\}$ and $\{\epsilon, \dot{\epsilon}\}$ to track reference or commanded trajectories, redundant-controlled are noninteracting controllers. The strumpletely decoupled, then $\tau_{\text {cart }}^{s}$ and $\tau_{\text {redun }}^{s}$ reveals that all $N$ actuated mane structure of the proposed composite controller control of the Cartesian- and the redulator joints contribute simultaneously to the 


\section{CONCLUSION AND FUTURE WORK}

The conceptual input relegation control philosophy [1] has been applied to the problem of resolving the kinematic redundancy of a serial-link manipulator as well as to dynamically modeling and controlling such a system. A new vector variable $\epsilon$ quantifying the redundant DOF was introduced and defined as linear combinations of the generalized velocities. The equations defining vector $\epsilon$ together with the kinematic velocity model yielded a well-specified solution for the generalized velocities. The general procedure was also used to resolve the redundancy at the acceleration level. A criteria for choosing $\epsilon$ and several approaches for selecting the $B(q)$ matrix were presented. In the first approach, $B$ was picked to be a constant matrix such that $\epsilon$ is a subset of the generalized velocities. This resulted in determining the inverse of the composite matrix $\left(J^{T}(g), B^{T}\right)^{T}$ analytically thus avoiding a numerical inversion. Furthermore, analytical and numerical methods for determining $B(q)$ such that it is orthogonal to the rows of the Jacobian matrix $J(q)$ were suggested, some of which applied to manipulators with multiple degrees of redundancy. The advantages of this were demonstrated through relegating the redundant DOF to produce minimum Euclidean norm solutions for the generalized velocities and accelerations, respectively. Additionally, a rigid body dynamical model consisting of two distinct sets of equations of motion was derived. One set is the Cartesian space equations of motio! governing the behavior of the nonredundant DOF. The other set governs the redundant DOF. A control architecture was suggested, which according to the separated form of the model, decouples the Cartesian- and redundant-DOF.

The research in this report has uncovered and identified a wealth of open research issues that warrant future attention. Vectors $\{\epsilon, \dot{\epsilon}\}$ were selected to minimize the Euclidean norm of the generalized velocities and accelerations, respectively. Clearly other criteria and analytical methods for selecting $\{\epsilon, \dot{\epsilon}\}$ need to be developed. The analytical methods for choosing $B(q)$ to be orthogonal to the rows of the Jacobian matrix in Section 3.3 need to be extended to allow for an arbitrary number of degrees of redundancy. The vector cross product method in Section 3.5 for deriving the orthogonal complement of the Jacobian allows for multiple degrees of redundancy. Unfortunately, the method is restricted to Jacobians having only two rows. Further research is needed to generalize this approach.

It has been shown in this report that the problems of resolving the kinematic redundancy of a serial link manipulator and of dynamically modeling a constrained mechanical system $[7,8,9]$ are intimately related. In view of this, another suggested future work area is to investigate the uses of the redundant DOF during hard contact motion of a serial-link redundant manipulator. Additionally, it is felt to be very worthwhile to combine the results presented here with those on modeling the constrained motion of a nonholonomic omnidirectional wheeled mobile platform $[15,20]$ to study the problems of kinematic redundancy and rigid body constraints in a combined mobility/manipulation system. 


\section{ACKNOWLEDGMENT}

This research was supported in part by the Office of Engineering Research Program, Basic Energy Sciences, U.S. Department of Energy, and in part by the Office of Technology Support Programs, Office of Nuclear Energy, U.S. Department of Energy, under Contract No. DE-AC05-84OR21400 with Martin Marietta Energy Systems, Inc. The author wishes to thank Dr. D. B. Reister and Dr. F. G. Pin for reviewing this manuscript and providing many insightful suggestions for its improvement. 


\section{APPENDIX A \\ THE JACOBIAN MATRIX FOR THE CESARm RESEARCH MANIPULATOR}

In this appendix the $(3 \times 4)$ Jacobian matrix which transforms the joint velocities of the lower four joints of the CESARm research manipulator $[6,10,11,12]$ to obtain the Cartesian translational velocities of the centerpoint of the wrist is specified. Let $j_{l m}$ denote the element located at the intersection of the $l$ th row and $m$ th column of $J(q)$. Let $c_{i}=\cos \left(q_{i}\right)$ and $s_{i}=\sin \left(q_{i}\right), i=1,2,3,4$. The elements are defined by:

$$
\begin{aligned}
j_{11}= & a_{4} s_{1} s_{2} s_{4}-a_{4}\left(c_{1} s_{3}+s_{1} c_{2} c_{3}\right) c_{4}-a_{3} c_{1} s_{3}-a_{3} s_{1} c_{2} c_{3} \\
& -d_{3} s_{1} s_{2}+d_{2} c_{1} \\
j_{12}= & -a_{4} c_{1} c_{2} s_{4}-a_{4} c_{1} s_{2} c_{3} c_{4}-a_{3} c_{1} s_{2} c_{3} \\
& +d_{3} c_{1} c_{2}, \\
j_{13}= & a_{4}\left(-c_{1} c_{2} s_{3}-s_{1} c_{3}\right) c_{4}-a_{3}\left(c_{1} c_{2} s_{3}+s_{1} c_{3}\right), \\
j_{14}= & -a_{4}\left(c_{1} c_{2} c_{3}-s_{1} s_{3}\right) s_{4}-a_{4} c_{1} s_{2} c_{4}, \\
j_{21}= & -a_{4} c_{1} s_{2} s_{4}+a_{4}\left(c_{1} c_{2} c_{3}-s_{1} s_{3}\right) c_{4}-a_{3} s_{1} s_{3}+a_{3} c_{1} c_{2} c_{3} \\
& +d_{3} c_{1} s_{2}+d_{2} s_{1}, \quad, \\
j_{22}= & -a_{4} s_{1} c_{2} s_{4}-a_{4} s_{1} s_{2} c_{3} c_{4}-a_{3} s_{1} s_{2} c_{3} \\
& +d_{3} s_{1} c_{2}, \\
j_{23}= & a_{4}\left(c_{1} c_{3}-s_{1} c_{2} s_{3}\right) c_{4}-a_{3}\left(s_{1} c_{2} s_{3}-c_{1} c_{3}\right) \\
j_{24}= & -a_{4}\left(c_{1} s_{3}+s_{1} c_{2} c_{3}\right) s_{4}-a_{4} s_{1} s_{2} c_{4}, \\
j_{31}= & 0 \\
j_{32}= & a_{4}\left(-s_{2} s_{4}+c_{2} c_{3} c_{4}\right)+a_{3} c_{2} c_{3} \\
& +d_{3} s_{2}, \\
j_{33}= & -a_{4} s_{2} s_{3} c_{4}-a_{3} s_{2} s_{3}, \\
j_{34}= & a_{4}\left(c_{2} c_{4}-s_{2} c_{3} s_{4}\right) \\
&
\end{aligned}
$$




\section{Appendix $A$}

where $\left[a_{3}, a_{4}, d_{2}, d_{3}\right]$ are constant Denavit-Hartenberg link length parameters which are illustrated in Fig. 2 and whose values are:

$$
\left[a_{3}, a_{4}, d_{2}, d_{3}\right]=[0.029,0.508,0.356,0.635](m)
$$




\section{APPENDIX B \\ EXTENSION TO MANIPULATORS \\ WITH TWO REDUNDANT DOF}

The problem is to extend the analytical method for selecting $B$ to specify a desired, reference value for the determinant of $\left(J^{T}, B^{T}\right)^{T}$ for manipulators containing two degrees of redundancy $(N-M=2)$. Let the $(2 \times N)$ matrix $B=\left(B_{1}^{T}, B_{2}^{T}\right)^{T}$, where $B_{i}=\left[b_{i 1}, b_{i 2}, \ldots, b_{i N}\right], i=1,2$.

Expanding along the last row of $\left(J^{T}(q), B^{T}(q)\right)^{T}$ using the Laplace expansion [13], the determinant of $\left(J^{T}(q), B^{T}(q)\right)^{T}$ is:

$$
\operatorname{det}\left[\begin{array}{c}
J(q) \\
B(q)
\end{array}\right]=B_{2}(q) \Delta
$$

where, here again, $\operatorname{det}[]$ denotes the determinant of [ ]. In Eq. (B.1), $\Delta\left(=\left[\Delta_{N 1}, \Delta_{N 2}, \ldots, \Delta_{N N}\right]^{T}\right)$ is the vector of cofactors, where $\Delta_{N i}$ is the cofactor of the $i$ th element of the $N$ th row of $\left(J^{T}(q), B^{T}(q)\right)^{T}$. Vector $\Delta$ is a function of the generalized coordinates and the elements of $B_{1}$. The scalar cofactor $\Delta_{N i}(i=1,2, \ldots, N)$ is defined by:

$$
\Delta_{N i}=(-1)^{N+i} B_{1}^{i} \Delta_{N-1}
$$

where ${ }^{i} \Delta_{N-1}$ is a $(N \times 1)$ vector defined by:

${ }^{i} \Delta_{N-1}=\left[{ }^{i} \Delta_{N-1,1},{ }^{i} \Delta_{N-1,2}, \ldots,{ }^{i} \Delta_{N-1, i-1}, 0,{ }^{i} \Delta_{N-1, i},{ }^{i} \Delta_{N-1, i+1}, \ldots,{ }^{i} \Delta_{N-1, N-1}\right]^{T}$

where ${ }^{i} \Delta_{N-1, k},(k=1,2, \ldots, N-1)$ is the cofactor of the $k$ th element of the $(N-1)$ th row of the $((N-1) \times(N-1))$ matrix contained within cofactor $\Delta_{N i}$. The $i$ th element of ${ }^{i} \Delta_{N-1}$ is zero.

Using the quantities defined above, the determinant of $\left(J^{T}(q), B^{T}(q)\right)^{T}$ can be expressed in a concise notation:

$$
\operatorname{det}\left[\begin{array}{c}
J(q) \\
B(q)
\end{array}\right]=B_{2} \Theta B_{1}^{T}
$$

where $\Theta$ is an $(N \times N)$ matrix defined by:

$$
\Theta=\left[\begin{array}{c}
{ }^{1} \Delta_{N-1}^{T}(-1)^{N+1} \\
{ }^{2} \Delta_{N-1}^{T}(-1)^{N+2} \\
\vdots \\
{ }^{N} \Delta_{N-1}^{T}(-1)^{N+N}
\end{array}\right]
$$


whose main diagonal elements are all zero. $\Theta$ is a function of the generalized coordinates and it will be shown by examples that it is a skew-symmetric matrix, i.e., $-\Theta=\Theta^{T}$.

The general class of $(N \times 2)$ orthogonal complements $\Sigma$ of $J$ is defined as the last two columns of $\left\{\left(J^{T}(q), B^{T}(q)\right)^{T}\right\}^{-1}$ :

$$
\Sigma(q)=\frac{\Theta}{B_{2} \Theta B_{1}^{T}}\left[-B_{2}^{T}, B_{1}^{T}\right]
$$

where a specific $\Sigma$ is obtained by the designer's choice of $B_{1}$ and $B_{2}$.

Suppose we select $B_{2}$ as:

$$
B_{2}=\left\{\Theta\left[\begin{array}{c}
1 \\
0_{((N-1) \times 1)}
\end{array}\right]\right\}^{T}=-{ }^{1} \Delta_{N-1}^{T}(-1)^{N+1} .
$$

If we select $B_{1}$ as:

$$
B_{1}=\left[\frac{1}{B_{2} B_{2}^{T}} \operatorname{det}^{*}\left[\begin{array}{l}
J(q) \\
B(q)
\end{array}\right], 0_{(1 \times(N-1))}\right]
$$

where $B_{2}$ is defined in Eq. (B.7) and where $\operatorname{det}^{*}\left[J^{T}(q), B^{T}(q)\right]^{T}$ is a constant, desired value of $\operatorname{det}\left[J^{T}(q), B^{T}(q)\right]^{T}$ selected by the designer, e.g., select $\operatorname{det}^{*}\left[J^{T}(q), B^{T}(q)\right]^{T} \neq 0$ to make $\left(J^{T}(q), B^{T}(q)\right)^{T}$ an invertible matrix. Then substituting Eqs. (B.7) and (B.8) into Eq. (B.4) yields:

$$
\operatorname{det}\left[\begin{array}{l}
J(q) \\
B(q)
\end{array}\right]=\operatorname{det}^{*}\left[\begin{array}{l}
J(q) \\
B(q)
\end{array}\right] .
$$

Given this choice of $B_{1}$ and $B_{2}$, the determinant of $\left[J^{T}(q), B^{T}(q)\right]^{T}$ is non-negative and can only be zero when vector ${ }^{1} \Delta_{N-1}=0_{(N \times 1)}$. Should all the components of ${ }^{1} \Delta_{N-1}$ be identically zero, $B_{2}$ may be selected as $-{ }^{i} \Delta_{N-1}^{T}(-1)^{N+i}$ for any $i(=2,3, \ldots, N)$ for which at least one of the components of ${ }^{i} \Delta_{N-1}^{T}$ (other than the $i$ th component) is nonzero. $B_{1}$ would then be selected as:

$$
B_{1}=\left[0_{(1 \times(i-1))}, \frac{1}{B_{2} B_{2}^{T}} \operatorname{det}^{*}\left[\begin{array}{l}
J(q) \\
B(q)
\end{array}\right], 0_{(1 \times(N-i))}\right] \text {. }
$$

An alternate analytical approach to picking $B$ so as to specify a desired reference value of $\operatorname{det}\left[J^{T}, B^{T}\right]^{T}$ is now discussed. It is assumed that the row vector $B_{2}$ has been selected to be linearly independent of the rows of $J$. Suppose $B_{1}^{T}$ is selected as:

$$
B_{1}^{T}=\frac{\Theta^{T} B_{2}^{T}}{B_{2} \Theta \Theta^{T} B_{2}^{T}} \operatorname{det}^{*}\left[\begin{array}{l}
J \\
B
\end{array}\right]=\frac{\Theta B_{2}^{T}}{B_{2} \Theta^{2} B_{2}^{T}} \operatorname{det}^{*}\left[\begin{array}{l}
J \\
B
\end{array}\right]
$$


where the skew-symmetry of $\Theta$ has been exploited. $\Theta^{2}$ is a symmetric matrix whose main diagonal elements are negative. Thus, the denominator of the right-hand side of Eq. (B.11) is a quadratic form [14]. Substituting Eq. (B.11) into Eq. (B.4) yields the solution for the determinant given by Eq. (B.9). Since $J \Sigma=0_{M \times 2}$, it is easy to see that $J B_{1}^{T}=0_{M \times 1}$.

The former method for determining matrix $B$ is now illustrated through two examples.

Example B1. Consider the problem of the determining matrix $B$ for the case of controlling a serial-link planar redundant manipulator with $N=4$ revolute joints. The configuration of the system is shown in Fig. 3. Only the Cartesian translational motion of the end effector is specified and controlled. Thus $M=2$ and the system has 2 redundant DOF. The composite matrix $\left(J^{T}, B^{T}\right)^{T}$ can be expanded to reveal its component elements:

$$
\left[\begin{array}{l}
J \\
B
\end{array}\right]=\left[\begin{array}{llll}
j_{11} & j_{12} & j_{13} & j_{14} \\
j_{21} & j_{22} & j_{23} & j_{24} \\
b_{11} & b_{12} & b_{13} & b_{14} \\
b_{21} & b_{22} & b_{23} & b_{24}
\end{array}\right]
$$

Applying Eq. (B.1) with $N=4$, the vector of cofactors $\Delta\left(=\left[\Delta_{41}, \Delta_{42}, \Delta_{43}, \Delta_{44}\right]^{T}\right)$ is defined by:

$$
\begin{aligned}
\Delta= & -\left|\begin{array}{lll}
j_{12} & j_{13} & j_{14} \\
j_{22} & j_{23} & j_{24} \\
b_{12} & b_{13} & b_{14}
\end{array}\right|,\left|\begin{array}{lll}
j_{11} & j_{13} & j_{14} \\
j_{21} & j_{23} & j_{24} \\
b_{11} & b_{13} & b_{14}
\end{array}\right| \\
& \left.-\left|\begin{array}{lll}
j_{11} & j_{12} & j_{14} \\
j_{21} & j_{22} & j_{24} \\
b_{11} & b_{12} & b_{14}
\end{array}\right|,\left|\begin{array}{lll}
j_{11} & j_{12} & j_{13} \\
j_{21} & j_{22} & j_{23} \\
b_{11} & b_{12} & b_{13}
\end{array}\right|\right]^{T}
\end{aligned}
$$

where $|\cdot|$ denotes the determinant of $\{\cdot\}$.

Applying the definition of the ${ }^{i} \Delta_{N-1}$ given by Eq. (B.3), we have:

$$
\begin{aligned}
& { }^{1} \Delta_{3}=\left[\begin{array}{lll} 
& 0 & \\
j_{13} j_{24} & - & j_{14} j_{23} \\
j_{14} j_{22} & - & j_{12} j_{24} \\
j_{12} j_{23} & - & j_{13} j_{22}
\end{array}\right] \\
& { }^{2} \Delta_{3}=\left[\begin{array}{lll}
j_{13} j_{24} & - & j_{14} j_{23} \\
j_{14} j_{21} & - & j_{11} j_{24} \\
j_{11} j_{23} & - & j_{13} j_{21}
\end{array}\right] \\
& { }^{3} \Delta_{3}=\left[\begin{array}{lll}
j_{12} j_{24} & - & j_{14} j_{22} \\
j_{14} j_{21} & - & j_{11} j_{24} \\
j_{11} j_{22} & - & j_{12} j_{21}
\end{array}\right]
\end{aligned}
$$




$$
{ }^{4} \Delta_{3}=\left[\begin{array}{lll}
j_{12} j_{23} & - & j_{13} j_{22} \\
j_{13} j_{21} & - & j_{11} j_{23} \\
j_{11} j_{22} & - & j_{12} j_{21}
\end{array}\right]
$$

Substituting Eqs. (B.14)-(B.17) into the right-hand side of Eq. (B.5) with $N=4$ gives the solution for $\Theta$ :

$$
\Theta=\left[\begin{array}{cccc}
0 & -j_{13} j_{24}+j_{14} j_{23} & -j_{14} j_{22}+j_{12} j_{24} & -j_{12} j_{23}+j_{13} j_{22} \\
j_{13} j_{24}-j_{14} j_{23} & 0 & j_{14} j_{21}-j_{11} j_{24} & j_{11} j_{23}-j_{13} j_{21} \\
-j_{12} j_{24}+j_{14} j_{22} & -j_{14} j_{21}+j_{11} j_{24} & 0 & -j_{11} j_{22}+j_{12} j_{21} \\
j_{12} j_{23}-j_{13} j_{22} & j_{13} j_{21}-j_{11} j_{23} & j_{11} j_{22}-j_{12} j_{21} & 0
\end{array}\right] .
$$

Equation (B.18) reveals that $\Theta$ is skew-symmetric. $B_{2}$ is given by:

$$
B_{2}=\left[0, j_{13} j_{24}-j_{14} j_{23}, j_{14} j_{22}-j_{12} j_{24}, j_{12} j_{23}-j_{13} j_{22}\right]
$$

in which Eq. (B.7) has been invoked with $N=4$. Given $\Theta$ and $B_{2}$ as defined by Eqs. (B.18) and (B.19), the quantities $B_{1}$, det $\left[J^{T}, B^{T}\right]^{T}$, and $\Sigma$ are obtained by using Eqs. (B.8), (B.4), and (B.6), respectively.

Example B2. Consider the problem of determining matrix $B$ for the case of controlling a serial-link planar redundant manipulator with $N=5$ revolute joints. Both the Cartesian translational and rotational motions of the end effector are specified and controlled. Thus $M=3$ and the system has 2 redundant DOF. The composite matrix $\left(J^{T}, B^{T}\right)^{T}$ can be expanded to reveal its component elements:

$$
\left[\begin{array}{l}
J \\
B
\end{array}\right]=\left[\begin{array}{lllll}
j_{11} & j_{12} & j_{13} & j_{14} & j_{15} \\
j_{21} & j_{22} & j_{23} & j_{24} & j_{25} \\
j_{31} & j_{32} & j_{33} & j_{34} & j_{35} \\
b_{11} & b_{12} & b_{13} & b_{14} & b_{15} \\
b_{21} & b_{22} & b_{23} & b_{24} & b_{25}
\end{array}\right]
$$

The $(5 \times 1)$ vectors ${ }^{i} \Delta_{4}(i=1,2, \ldots, 5)$ are obtained by applying Eq. (B.3) with $N=5$ : 
${ }^{1} \Delta_{4}=\left[\begin{array}{ccc}0 & \\ -\left|\begin{array}{lll}j_{13}, & j_{14}, & j_{15} \\ j_{23}, & j_{24}, & j_{25} \\ j_{33}, & j_{34}, & j_{35} \\ j_{12}, & j_{14}, & j_{15} \\ j_{22}, & j_{24}, & j_{25} \\ j_{32}, & j_{34}, & j_{35}\end{array}\right| \\ -\left|\begin{array}{lll}j_{12}, & j_{13}, & j_{15} \\ j_{22}, & j_{23}, & j_{25} \\ j_{32}, & j_{33}, & j_{35}\end{array}\right| \\ j_{12}, & j_{13}, & j_{14} \\ j_{22}, & j_{23}, & j_{24} \\ j_{32}, & j_{33}, & j_{34}\end{array} \mid\right]$

(B.21)

${ }^{2} \Delta_{4}=\left[\begin{array}{c}-\left|\begin{array}{lll}j_{13}, & j_{14}, & j_{15} \\ j_{23}, & j_{24}, & j_{25} \\ j_{33}, & j_{34}, & j_{35}\end{array}\right| \\ 0 \\ -\left|\begin{array}{lll}j_{11}, & j_{14}, & j_{15} \\ j_{21}, & j_{24}, & j_{25} \\ j_{31}, & j_{34}, & j_{35}\end{array}\right| \\ -\left|\begin{array}{lll}j_{11}, & j_{13}, & j_{15} \\ j_{21}, & j_{23}, & j_{25} \\ j_{31}, & j_{33}, & j_{35}\end{array}\right| \\ \begin{array}{lll}j_{11}, & j_{13}, & j_{14} \\ j_{21}, & j_{23}, & j_{24} \\ j_{31}, & j_{33}, & j_{34}\end{array} \mid\end{array}\right]$,

(B.22) 
36 Appendix B

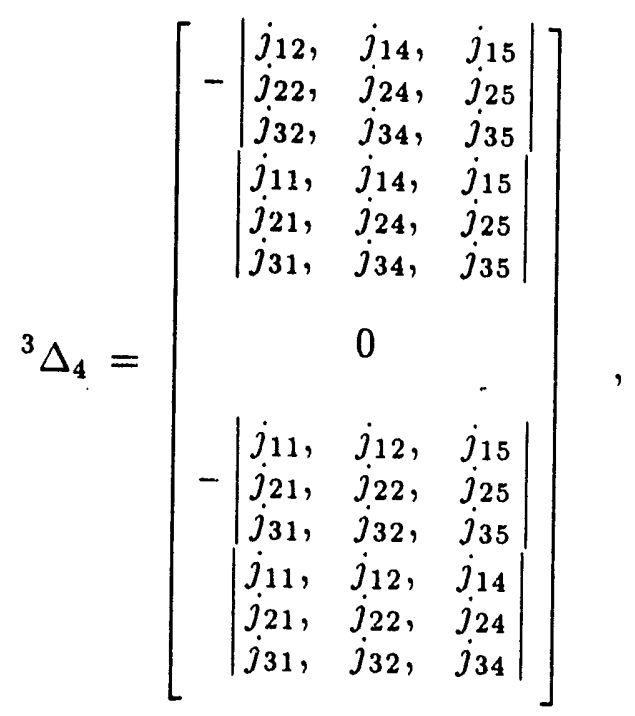

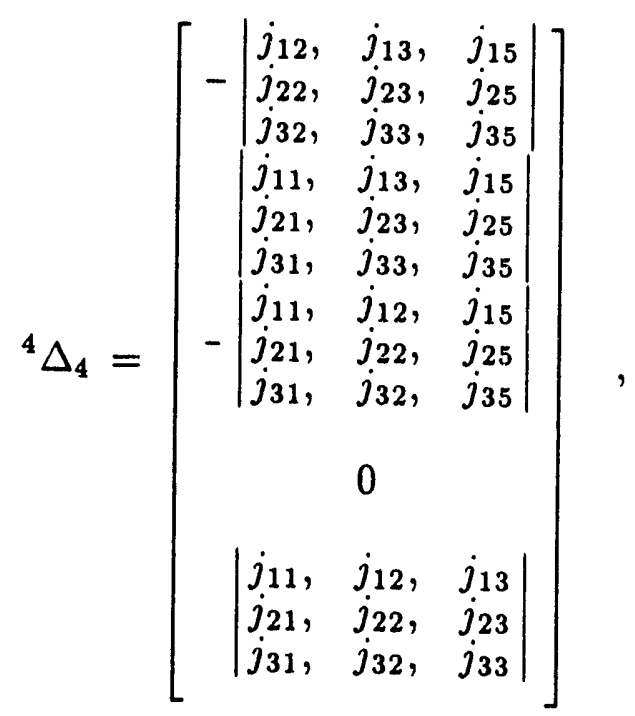




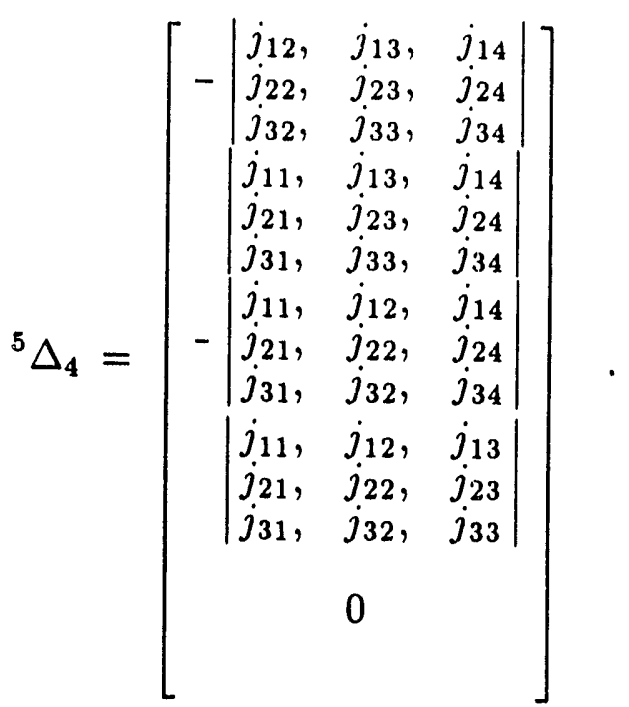

Substituting Eqs. (B.21)-(B.25) into the right-hand side of Eq. (B.5) with $N=5$, it is easy to see that $\Theta$ is skew-symmetric. $B_{2}$ is given by:

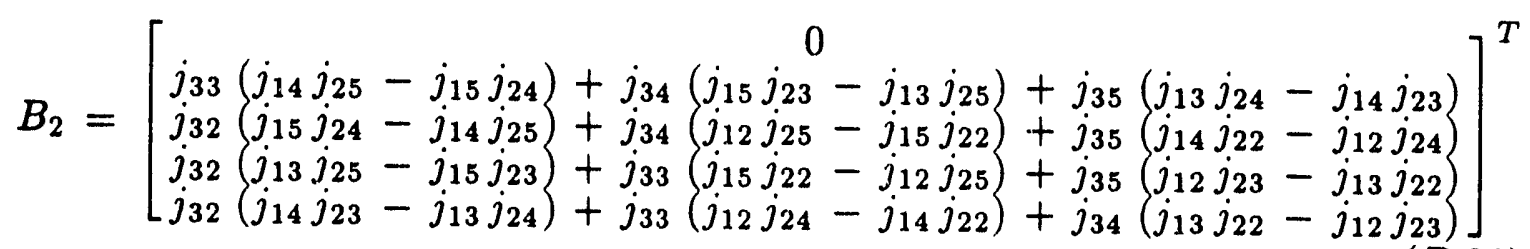

in which Eq. (B.7) has been invoked. Vector $B_{1}$ can be determined from Eq. (B.8). 


\section{APPENDIX C \\ DETERMINING SINGULARITY \\ AND RANK OF MATRIX $\left(J^{T} J\right)$}

In this appendix it will be proven by analytical techniques that matrix $\left(J^{T} J\right)$ has less that full rank and thus is singular. The matrix relations given by Eq. (5) will be used in evaluating the rank of $\left(J^{T} J\right)$. Additionally, the following matrix property will be used. Two $(n \times n)$ real matrices $\mathrm{X}$ and $\mathrm{Y}$ are given. Matrix $\mathrm{X}$ is assumed to possess full rank $n$ and thus is nonsingular. Then the following mathematical relation applies [13]:

$$
\operatorname{rank}(Y)=\operatorname{rank}(X Y)=\operatorname{rank}(Y X)=\operatorname{rank}\left(X^{T} Y\right)=\operatorname{rank}\left(Y X^{T}\right) .
$$

The rank of $\left(J^{T} J\right)$ may now be determined. By the property of Eq. (C.1), the following relation holds:

$$
\operatorname{rank}\left(J^{T} J\right)=\operatorname{rank}\left\{\left[\begin{array}{c}
\Pi^{T} \\
\Sigma^{T}
\end{array}\right] J^{T} J\left[J^{T}, B^{T}\right]\right\} .
$$

The rank of the $(N \times N)$ matrix within the braces on the right-hand side of Eq. (C.2) is now analyzed by first carrying out the multiplications:

$$
\operatorname{rank}\left(J^{T} J\right)=\operatorname{rank}\left\{\left[\begin{array}{ll}
\Pi^{T} J^{T} J J^{T}, & \Pi^{T} J^{T} J B^{T} \\
\Sigma^{T} J^{T} J J^{T}, & \Sigma^{T} J^{T} J B^{T}
\end{array}\right]\right\} .
$$

The right-hand side of Eq. (C.3) is simplified by invoking the matrix relations in Eq. (5):

$$
\operatorname{rank}\left(J^{T} J\right)=\operatorname{rank}\left\{\left[\begin{array}{cc}
J J^{T} & J B^{T} \\
0_{((N-M) \times M)} & 0_{((N-M) \times(N-M))}
\end{array}\right]\right\} .
$$

Equation (C.4) reveals that the last $(N-M)$ rows of matrix $\left([\Pi, \Sigma]^{T} J^{T} J\left[J^{T}, B^{T}\right]\right)$ contain all zeros. Thus matrix $\left([\Pi, \Sigma]^{T} J^{T} J\left[J^{T}, B^{T}\right]\right)$ is singular. Furthermore, the $\operatorname{rank}\left([\Pi, \Sigma]^{T} J^{T} J\left[J^{T}, B^{T}\right]\right)=M$. It should be mentioned that the upper $M$ rows on the right-hand side of Eq. (C.4) form the matrix $\left(J\left[J^{T}, B^{T}\right]\right)$, which has $\operatorname{rank} M$ because the rank of $J(=M)$ is unchanged by postmultiplying it by the $(N \times N)$ nonsingular matrix $\left[J^{T}, B^{T}\right][13]$.

Since matrix $\left([\Pi, \Sigma]^{T} J^{T} J\left[J^{T}, B^{T}\right]\right)$ is singular, then matrix $\left(J^{T} J\right)$ is also singular with $\operatorname{rank}\left(J^{T} J\right)=M$ because the ranks of these two matrices are the same as shown in Eq. (C.2). It should be mentioned that matrices $\left(J J^{T}\right)$ and $\left(J^{T} J\right)$ have the same eigenvalues, except that $\left(J^{T} J\right)$ has $(N-M)$ extra zero valued eigenvalues [14], which is readily confirmed by Eq. (C.4). 


\section{REFERENCES}

1. H. Hemami and H. D. Ong, "Philosophy, Structure, and Examples of Relegated Control," J. of Intelligent and Robotic Systems 2, 53-72, 1989.

2. D. N. Nenchev, "Redundancy Resolution through Local Optimization: A Review," J. of Robotic Systems 6(6), 769-798, December 1989.

3. B. Siciliano, "Kinematic Control of Redundant Robot Manipulators: A Tutorial," J. of Intelligent and Robotic Systems 3, 201-212, 1990.

4. J. Baillieul and D. P. Martin, "Resolution of Kinematic Redundancy" in Robotics, Proceedings of Symposia in Applied Mathematics 41, 49-90, Louisville, Kentucky, January 1990 (American Mathematical Society Short Course Lecture Notes).

5. P. Belmans and J.-C. Culioli, "A New Approach to Solve the Kinematics Resolution of a Redundant Robot," Oak Ridge National Laboratory Technical Report ORNL/TM-11435 CESAR-89/53, March 1990.

6. R. V. Dubey, J. A. Euler, and S. M. Babcock, "Real-Time Implementation of an Optimization Scheme for Seven-Degree-of-Freedom Redundant Manipulators," IEEE Trans. on Robotics and Automation 7(5), 579-588, October 1991.

7. F. R. Gantmacher, Lectures in Analytical Mechanics, USSR: Mir Publishers, 1975, Chapter 1, Section 10.

8. H. Hemami, "A Feedback On-Off Model of Biped Dynamics," IEEE Trans. on Systems, Man, and Cybernetics SMC-10(7), 376-383, July 1980, Section IV.

9. R. K. Kankaanranta and H. N. Koivo, "Dynamics and Simulation of Compliant Motion of a Manipulator," IEEE J. Robotics and Automation 4(2), 163-173, April 1988.

10. F. G. Pin, M. Beckerman, P. F. Spelt, J. T. Robinson, and C. R. Weisbin, "Autonomous Mobile Robot Research Using the HERMIES-III Robot," Proceedings of the IEEE/RSJ International Workshop on Intelligent Robots and Systems '89, September 4-6, 1989, Tsukuba, Japan, 251-256.

11. C. R. Weisbin, B. L. Burks, J. R. Einstein, R. R. Feezell, W. W. Manges, and D. H. Thompson, "HERMIES-III: A Step Toward Autonomous Mobility, Manipulation and Perception," Robotica 8, Part 1, January-March 1990, 7-12.

12. J. N. Anderson and W. D. Combs, "Link Mass Properties of the CESAR Research Manipulator," Martin Marietta Energy Systems, Inc., Technical Report No. MCTR-0987-14, September 1987.

13. F. R. Gantmacher, The Theory of Matrices 1, Chelsea Publishers, 1960.

14. B. Noble and J. W. Daniel, Applied Linear Algebra, 2nd Ed., Prentice-Hall, 1977, page 297.

15. D. B. Reister and M. A. Unseren, "Position and Force Control of a Vehicle with Two or More Steerable Drive Wheels," IEEE Transactions on Robotics and Automation (submitted).

16. W. C. Walton, Jr. and E. C. Steeves, "A New Matrix Theorem and Its Application for Establishing Independent Coordinates for Complex Dynamical Systems With Constraints," NASA Technical Report TR R-326, 1969. 
17. J. W. Kamman and R. L. Huston, "Dynamics of Constrained Multibody Systems," ASME J. of Applied Mechanics 51, 899-903, December 1984.

18. H. Goldstein, Classical Mechanics, 2nd Ed., Addison-Wesley, 1980, Chapters 4, 5 , and 6.

19. W. H. Press, B. P. Flannery, S. A. Teukolsky, and W. T. Vetterling, Numerical Recipes in C, The Art of Scientific Computing, Cambridge University Press, 1988, Chapter 11.

20. D. B. Reister, "A New Wheel Control System for the Omnidirectional HERMIES-III Robot," Robotica 10, 1992, 351-360. 


\section{INTERNAL DISTRIBUTION}

ORNL/TM-12165

1. B. R. Appleton

2. J. E. Baker

3. A. L. Bangs

4. M. Beckerman

5. J. F. Jansen

6. J. P. Jones

7. H. E. Knee

8. R. L. Kress

9-13. R. C. Mann

14-18. F. G. Pin

19. V. Protopopescu

20. K. Rahmani

21. D. B. Reister

22. P. F. Spelt

23. T. Steinel
24. F. J. Sweeney

25. E. C. Uberbacher

26-30. M. A. Unseren

31. R. C. Ward

32. Y. Watanabe

33. EP\&MD Reports Office

34-35. Laboratory Records Department

36. Laboratory Records, ORNL-RC

37. Document Reference Section

38. Central Research Library

39. ORNL Patent Section

\section{EXTERNAL DISTRIBUTION}

40. Office of Assistant Manager, Energy Research and Development, Department of Energy, Oak Ridge Operations, Oak Ridge, TN 37831

41. Dr. Peter Allen, Department of Computer Science, 450 Computer Science, Columbia University, New York, NY 10027

42. Dr. Richard Becker, 2780 Oak Ridge Turnpike, Oak Ridge, TN 37830

43. Dr. Wayne Book, Department of Mechanical Engineering, J. S. Coon Building, Room 306, Georgia Institute of Technology, Atlanta, GA 30332

44. Professor Roger W. Brockett, Wang Professor of Electrical Engineering and Computer Science, Division of Applied Sciences, Harvard University, Cambridge, MA 02138

45. Professor John J. Dorning, Department of Nuclear Engineering and Physics, Thornton Hall, McCormick Rd., University of Virginia, Charlottesville, VA 22901

46. Dr. Steven Dubowsky, Massachusetts Institute of Technology, Building 3, Room 469A, 77 Massachusetts Ave., Cambridge, MA 02139

47. Dr. Avi Kak, Robot Vision Lab, Department of Electrical Engineering, Purdue University, Northwestern Ave., Engineering Mall, West Lafayette, IN 47907

48. Dr. James E. Leiss, 13013 Chestnut Oak Dr., Gaithersburg, MD 20878

49. Dr. Oscar P. Manley, Division of Engineering, Mathematical, and Geosciences, Office of Basic Energy Sciences, ER-15, U.S. Department of Energy - Germantown, Washington, DC 20545

50. Professor Neville Moray, Department of Mechanical and Industrial Engineering, University of Illinois, 1206 West Green St., Urbana, IL 61801

51. Dr. Wes Snyder, Department of Radiology, Bowman Gray School of Medicine, 3005 Hawthorne Dr., Winston-Salem, NC 27103

52. D. Tesar, Department of Mechanical Engineering, University of Texas, 26 San Jacinto, Austin, TX 78712

53. Professor Mary F. Wheeler, Rice University, Department of Mathematical Sciences, P.O. Box 1892, Houston, TX 77251

54-63. Office of Scientific and Technical Information, P.O. Box 62, Oak Ridge, TN 37831 
DATE FILMED $12 / 9 / 92$ 
